\title{
Biotransport kinetics and intratumoral biodistribution of malonodiserinolamide- derivatized [60]fullerene in a murine model of breast adenocarcinoma
}

\author{
This article was published in the following Dove Press journal: \\ International Journal of Nanomedicine \\ 15 November 2017 \\ Number of times this article has been viewed
}

\author{
Norman A Lapin' \\ Leoncio A Vergara ${ }^{1,2}$ \\ Yuri Mackeyev ${ }^{3,4}$ \\ Jared M Newton ${ }^{1,5}$ \\ Sean A Dilliard ${ }^{6}$ \\ Lon J Wilson ${ }^{3,4}$ \\ Steven A Curley' \\ Rita E Serda ${ }^{1,7}$ \\ 'Michael E DeBakey Department of \\ Surgery, Baylor College of Medicine, \\ ${ }^{2}$ Institute of Biosciences \& Technology, \\ Texas A\&M University, ${ }^{3}$ Department \\ of Chemistry, Rice University, \\ ${ }^{4}$ The Smalley-Curl Institute for \\ Nanoscale Science and Technology, \\ Rice University, ${ }^{5}$ Interdepartmental \\ Program in Translational Biology \\ and Molecular Medicine, Baylor \\ College of Medicine, ${ }^{6}$ Department \\ of Chemical and Biomolecular \\ Engineering, Rice University, Houston, \\ TX, ${ }^{7}$ Department of Chemical and \\ Biological Engineering, University of \\ New Mexico, Albuquerque, NM, USA
}

\begin{abstract}
Fullerene is a highly versatile nanoparticle (NP) platform for drug delivery to sites of pathology owing to its small size and both ease and versatility of chemical functionalization, facilitating multisite drug conjugation, drug targeting, and modulation of its physicochemical properties. The prominent and well-characterized role of the enhanced permeation and retention (EPR) effect in facilitating NP delivery to tumors motivated us to explore vascular transport kinetics of a water-soluble [60]fullerene derivatives using intravital microscopy in an immune competent murine model of breast adenocarcinoma. Herein, we present a novel local and global image analysis of vascular transport kinetics at the level of individual tumor blood vessels on the micron scale and across whole images, respectively. Similar to larger nanomaterials, [60]fullerenes displayed rapid extravasation from tumor vasculature, distinct from that in normal microvasculature. Temporal heterogeneity in fullerene delivery to tumors was observed, demonstrating the issue of nonuniform delivery beyond spatial dimensions. Trends in local region analysis of fullerene biokinetics by fluorescence quantification were in agreement with global image analysis. Further analysis of intratumoral vascular clearance rates suggested a possible enhanced penetration and retention effect of the fullerene compared to a $70 \mathrm{kDa}$ vascular tracer. Overall, this study demonstrates the feasibility of tracking and quantifying the delivery kinetics and intratumoral biodistribution of fullerene-based drug delivery platforms, consistent with the EPR effect on short timescales and passive transport to tumors.
\end{abstract}

Keywords: fullerene, intravital microscopy, breast cancer, enhanced permeation and retention, drug delivery

\section{Introduction}

[60]Fullerenes, first prepared from graphite vaporization by laser irradiation in $1985^{1}$ and later scaled-up for chemical modification, ${ }^{2}$ are spherical cages composed of 60 carbon atoms with a diameter of $<1 \mathrm{~nm}$ and can be classified as the smallest of nanoparticles (NPs). For $>25$ years, derivatives of this class of NP have served as a platform for a variety of biomedical applications including X-ray and magnetic resonance imaging (MRI) contrast agents, ${ }^{3-6}$ drug delivery, ${ }^{5-13}$ antioxidant neuroprotection, ${ }^{14,15}$ and free radical formation for cancer cytotoxicity with ${ }^{16-18}$ and without ${ }^{19}$ [60]fullerene photoexcitation. The small size of [60]fullerene derivatives makes them ideal candidates for drug delivery, potentially offering an increased penetration of tissues and rapid cellular uptake ${ }^{20-25}$ with minimal toxicity to normal cells. ${ }^{8,18,19,26,27}$ Owing to their spheroidal shape, [60]fullerenes have been functionalized at as many as 48 locations
Correspondence: Norman A Lapin Michael E DeBakey Department of Surgery, Baylor College of Medicine, One Baylor Plaza, Houston, TX 77030, USA

Tel +I 7I3 7987733

Email norman.lapin@bcm.edu $\mathrm{BY}$
$\mathrm{NC}$ and incorporate the Creative Commons Attribution - Non Commercial (unported, v3.0) License (http://(creativecommons.org/licenses/lby-nc/3.0/). By accessing the work you for commercial use of this work, please see paragraphs 4.2 and 5 of our Terms (https://www.dovepress.com/terms.php). 
on a single $\mathrm{NP}^{28}$ and even branched at individual derivative locations to provide multisite drug attachment per [60]fullerene NP, ${ }^{8}$ potentially boosting therapeutic dose at lower NP concentration. ${ }^{3}$ Hence, [60]fullerene is a highly flexible NP platform that is potentially a drug delivery vector and dosetunable depending on the therapeutic application.

One such application of [60]fullerene derivatives is in the treatment of solid malignant tumors. ${ }^{9,11-13}$ However, there are numerous biological barriers that must be overcome for efficacious delivery of these NPs to the tumor site all of which depend on NP solubility in each biological compartment. These transport barriers include uptake by the mononuclear phagocyte system (MPS), systemic clearance, tumor vasculature extravasation, interstitial transport in cancerous tissues, and tumor cell uptake. Furthermore, to activate the bioavailability of this hydrophobic fullerene platform, it is necessary to chemically modify it by the attachment of polar functional groups. ${ }^{8}$ Additional obstacles to tumor drug and NP delivery include high tumor interstitial fluidic pressure, ${ }^{29}$ intratumoral variability in blood vessel diameter, permeability, number density, and volumetric distribution, ${ }^{30,31}$ all of which cause highly heterogeneous distribution of drug and NP in the tumor. ${ }^{32}$

Despite the promise of water-soluble fullerene derivatives as drug delivery vectors, there have been a limited number of investigations of the biokinetics of water-soluble fullerene derivatives. At the cellular level, investigators demonstrated that upon cellular uptake of PromoFluor-633-conjugated malonodiserinolamide-derivatized [60]fullerene $\left(\mathrm{C}_{60}\right.$-serPF) by liver cancer cells and HeLa cells in vitro, aggregates of $\mathrm{C}_{60}$-serPF were found in the nuclei of those cells ${ }^{21,25}$ and that for liver cancer cells, the uptake was molecular pathway dependent. ${ }^{21} \mathrm{C}_{60}$-serPF uptake and biodistribution was further studied in mice, and this agent was found to predominantly accumulate in the kidney and liver as well as in hepatic tumors on the scale of hours. ${ }^{21}$ Toxicity studies show that some types of water-soluble fullerene derivatives conjugated to antineoplastic agents can be toxic to both normal cells and cancer cells, ${ }^{8}$ and solubility is tunable such that increases in solubility negate their toxicity by up to seven orders of magnitude. ${ }^{19}$ Malonodiserinolamide-derivatized [60]fullerene $\left(\mathrm{C}_{60}\right.$-ser) itself (consisting of a hydrophobic core surrounded symmetrically by hydrophilic serinol groups) exhibits low cytotoxicity to both normal and cancer cells yet demonstrates cytotoxicity when covalently modified with the chemotherapeutic, Taxol ${ }^{\circledR}$ (paclitaxel). ${ }^{8}$

To gain further insight into the behavior and biokinetics of water-soluble fullerene derivatives in vivo, real-time dynamic information is needed beyond what endpoint analyses can offer. Such time-resolved studies can provide specific information about the temporal mechanisms behind intratumoral barriers to drug and NP delivery and related heterogeneity that lead to poor or nonuniform intratumoral drug and NP biodistribution. ${ }^{33}$ Intravital microscopy (IVM) is a powerful tool that can provide detailed information about tumor and drug or NP interactions over time and hence supplement the missing temporal information. IVM applies the optical sectioning power of confocal microscopy to image the real-time delivery of fluorescently labeled drugs and NPs in the microvasculature of live animals with micron scale resolution.

In this study, we perform continuous quantification of fullerene dynamics at a temporal resolution of $\sim 1 \mathrm{fps}$ across micron-scale spatially resolved intra- and extravascular regions. Specifically, we employ IVM to investigate the intratumoral biotransport kinetics of $\mathrm{C}_{60}$-ser delivered intravenously to the microvasculature of subcutaneously allografted 4T1-luc breast cancer tumors versus normal tissue in live mice. Conjugation of $\mathrm{C}_{60}$-ser with the fluorescent dye PromoFluoro-633 ( $\mathrm{C}_{60}$-serPF) enabled fluorescence-based tracking and relative quantification of $\mathrm{C}_{60}$-ser in vivo. As this fluorophore $(\sim 650 \mathrm{Da})$ is comparable in size and structure to small molecule cancer drugs such as paclitaxel (854 Da), it can further be considered sterically as a model drug for the study of $\mathrm{C}_{60}$-serPF as a drug carrier. With high spatial resolution and time-resolved confocal microscopy imaging, quantitative analysis of the biotransport kinetics involved in the microvascular delivery, extravasation, and tumor interstitial transport of $\mathrm{C}_{60}$-serPF were conducted both globally across video images and spatially localized at specific intravascular regions of interest (iROIs) and extravascular regions of interest (eROIs) corresponding to regions in and around individual blood vessel segments. In developing these methods of spatiotemporal quantification of real-time in vivo biodynamics, our investigation is best regarded as a pilot study for such a framework.

To our knowledge, no intravital biokinetic studies of any fullerene derivative have previously been reported. Furthermore, while many IVM studies quantitatively monitor drug or NP kinetics with temporal resolution on the order of minutes to hours ${ }^{34-37}$ and even days, ${ }^{35}$ no study has quantitatively tracked initial NP or drug delivery influx dynamics in the tumor microenvironment at a resolution on the timescale of seconds. $\mathrm{As}_{60}$-serPF clears rapidly from the blood stream (as evidenced by the color change of mouse urine occurring within $15{\text { min of } \mathrm{C}_{60} \text {-serPF injection }}^{21}$ ), capture of $\mathrm{C}_{60}$-serPF 
dynamics within the first several minutes postinjection at high temporal resolution is critical to the observation of its biokinetic behavior. Herein, we demonstrate that the water-soluble fullerene derivative $\mathrm{C}_{60}$-serPF exhibits rapid extravasation from vessels within the tumor microenvironment, capturing the biokinetics of these NPs by IVM with temporal resolution on the scale of a second. With respect to NP and drug delivery, various IVM studies have used a variety of methods to partition intravascular and extravascular regions over the course of imaging including image segmentation, ${ }^{37-39}$ selection of specific regions of interest (ROIs) within video field of view (FOV), ${ }^{31,34}$ or intensity profiles across regions. ${ }^{37,40}$ However, while these studies have performed global analyses across the entire image $\mathrm{FOV}^{31}$ and/or combined data from multiple specific ROIs, ${ }^{34}$ to our knowledge, no studies have explicitly tracked local extravasation dynamics from specific blood vessels as we do in this study.

To better understand the solution phase behavior of $\mathrm{C}_{60}$-ser, we conducted ex vivo characterization of $\mathrm{C}_{60}$-ser physicochemical properties including dynamic light scattering (DLS), zeta potential measurements, and scanning electron microscopy (SEM). We found that $\mathrm{C}_{60}$-ser molecules may exist as both aggregates and free NPs, where aggregate geometrical arrangement is a function of hydrophilic and hydrophobic parts of the $\mathrm{C}_{60}$-serPF molecule, giving the NP special aggregation properties.

\section{Methods}

\section{Synthesis and characterization of molecular agents and dyes}

Malonodiserinolamide-derivatized [60]fullerene $\left(\mathrm{C}_{60}\right.$-ser, $2.21 \mathrm{kDa}$ ) was prepared as previously described. ${ }^{41} \mathrm{C}_{60}$-serPF $(2.87 \mathrm{kDa})$, formed by the covalent attachment of fluorescent dye, PromoFluor-633 (PromoCell GmbH, Heidelberg, Germany) to $\mathrm{C}_{60}$-ser, was synthesized and purified according to the procedure described in Raoof et al. ${ }^{21}$ Fluorescein isothiocyanate (FITC)-conjugated dextran (FITC-dextran; $70 \mathrm{kDa}$ ) purchased from Thermo Fisher Scientific (Waltham, MA, USA) served as a nonextravasating blood vessel tracer with peak excitation at $488 \mathrm{~nm}$ and emission in the FITC channel $(525 \pm 25 \mathrm{~nm})$. PromoFluor-633 of $\mathrm{C}_{60}$-serPF has peak excita-

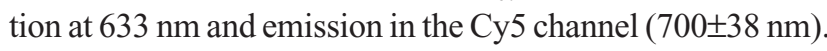
High performance liquid chromatography (HPLC) data of Figure S1 validate that this compound is present in high quantity relative to other isomers. The HPLC data further verifies that the covalently bound PromoFluor-633 dye remains attached to $\mathrm{C}_{60}$-serPF in aqueous and biological environments and that no free dye is present with the $\mathrm{C}_{60}-$ serPF NP.
Characterization of $\mathrm{C}_{60}$-ser included dynamic light scattering (DLS), zeta potential, and scanning electron microscopy (SEM) measurements. DLS and zeta potential measurements were performed on a Malvern Zetasizer Nano (Malvern Instruments, Malvern, UK). To collect DLS and zeta potential readings over time, $\mathrm{C}_{60}$-ser was dissolved in an aqueous solvent (as stated), agitated briefly, and placed immediately in a cuvette and into the Zetasizer where it remained for the duration of readings up to $66 \mathrm{~h}$. Measurements of $\mathrm{C}_{60}$-serPF are not included here due to inability of the Zetasizer to obtain consistently stable readings. This limitation is likely due to interference between the PromoFluor-633 emission spectrum and the frequency of the Zetasizer laser used to collect DLS (633 nm), which overlap.

To collect SEM images of $\mathrm{C}_{60}$-ser, a solution of $10 \mathrm{mg} / \mathrm{mL}$ $\mathrm{C}_{60}$-ser in deionized water (DIW) was lyophilized and subsequently coated onto a 300-mesh transmission electron microscopy (TEM) grid.

Images were acquired using a Hitachi SU8230 scanning electron microscope (Hitachi High-Technologies, Hillsboro, OR, USA) equipped with a secondary electron detector.

\section{Animal model}

Female BALB/c mice purchased from Harlan Sprague Dawley, Inc. (Houston, TX, USA) were housed in standard temperature and lighting conditions with free access to food and water. Acclimated mice were injected subcutaneously with 4T1-luc breast cancer cells $\left(5 \times 10^{5}\right.$ cells $/ 50 \mu \mathrm{L}$ PBS; Caliper Life Sciences, Hopkinton, MA, USA) into the left abdominal wall to establish allograft breast tumors. When tumors reached a size of $\sim 10 \mathrm{~mm}$ in diameter, mice were selected for IVM experiments. All animal procedures were performed in accordance with protocols approved by the Institutional Animal Care and Use Committee at the Baylor College of Medicine and in accordance with the 8th edition of the Guide for the Care and Use of Laboratory Animals (NRC, 1996).

\section{IVM}

IVM was used to monitor the transport kinetics of $\mathrm{C}_{60}$-ser in vivo delivered to subcutaneous 4T1 breast cancer tumors in a murine animal model. All animal experiments performed in this study were end-point terminal experiments, involving nonsurvival surgery. Animals were anesthetized by the inhalation of isoflurane in oxygen to a deep plane of anesthesia. The anesthesia level was subsequently maintained by the adjustment of isoflurane concentration from 2 to $3 \%$. Animals were then secured in the supine position on a heated plate (to maintain body temperature throughout the entire experiment) and a midline abdominal incision was made, 
followed by a careful separation of abdominal cutaneous layers with a cotton swab to form a skin flap, exposing the tumor and blood vessels growing in the flap. The skin flap was then affixed to the surface of a rubber pad support. The preparation was next transferred to the microscope stage and maintained moist by periodic irrigation with PBS. To allow high resolution imaging with water immersion lenses and to reduce motion, a coverslip was set on top of the samples using a micro-positioning device.

A Nikon A1R laser scanning confocal microscope (Nikon Instruments, Melville, NY, USA) fitted with a waterimmersion objective lens (CFI75 LWD 16× W, 0.8NA) was used to collect time lapse images of tumor microvasculature and local microenvironment of 4T1 breast adenocarcinoma in live mice. Once tumor microvasculature was in focus in bright field, the microscope was switched to confocal mode and image capture was performed at $0.5 \mathrm{fps}$ in either Galvano (high resolution) or Resonant (high speed) image collection mode. A bolus dose of $20-50 \mu \mathrm{L}$ of $70 \mathrm{kDa}$ FITC-dextran, too large to extravasate appreciably $(10 \mathrm{mg} / \mathrm{mL})$, and an equal volume of $\mathrm{C}_{60}$-serPF $(2 \mathrm{mg} / \mathrm{mL})$ were injected intravenously via retro-orbital injection. These molecular agents were coinjected in the tumor-bearing mice, while in the normal mouse, FITC-dextran was injected prior to $\mathrm{C}_{60}$-serPF. Imaging commenced just prior to the injection of $\mathrm{C}_{60}$-serPF (with or without coinjection of FITC-dextran) and continued until fluorescence changes appeared to stabilize ( $\sim 2 \mathrm{~min}$ after $\mathrm{C}_{60}$-serPF injection). Vascular delivery, extravasation, and interstitial (ie, extravascular and extracellular) transport of $\mathrm{C}_{60}$-serPF in the tumor microenvironment were imaged continuously in real time. At the termination of the imaging session, animals were euthanized in accordance with approved protocols.

\section{Image analysis}

Local ROIs were selected from each time-lapse video to quantify representative extravasation dynamics from individual blood vessel regions. Global ROIs were created across the entire FOV for all frames in each video to capture global extravasation and extravascular transport dynamics. Relative changes in fluorescence intensity were assumed to be proportional to changes in NP concentrations.

Note that where fluorescence background intensity is high (eg, as for mouse \#2), the pattern of intensity increase in the diffusing NP still follows that expected for intravascular transport and extravascular diffusion across a barrier. These dynamics are typically characterized by a rapid influx to the intravascular region (observed in both local and global ROIs) followed immediately by increases in extravascular ROIs adjacent to intravascular ROIs and subsequently for
iROIs (in local ROI analysis only) that are further still from the iROI where NP influx was initially tracked.

\section{Selection of local ROIs}

The topology of the tumor microenvironment, characterized by a high density of blood vessels in three-dimensional space, presents a challenge for the quantitative analysis of intratumoral dynamics and biodistribution. Quantification of NP extravasation is especially challenging because of the multidirectional superposed regions of influx of NPs into the tumor interstitial region. A number of IVM studies have approached fluorescence quantification of intratumoral NP dynamics selecting ROIs through 2D and 3D image segmentations (eg, thresholding) of features including blood vessels, cancer cells, and tumor-associated macrophages, ${ }^{37-39} \mathrm{NP}$ counts in intra- and extravascular regions ${ }^{42}$ and by selecting specific $\mathrm{ROIs}^{34}$ or intensity profiles ${ }^{37,40}$ within image frames. With ROIs defined as such, these investigators have studied tumor intravascular dynamics, extravasation, and interstitial and intracellular accumulation of NPs. However, error in the quantification of NP transport kinetics and accumulation may arise from factors including tissue autofluorescence, movement of vascular structures between image frames, and extraneous NP fluorescence in ROIs. Such extraneous fluorescence may arise from the fluorescence of neighboring blood vessels in adjacent optical planes, as well as extravasation may arise from blood vessels that have not been properly taken into account.

In this study, fullerene extravasation dynamics were assessed in local micron-scale ROIs through the selection of iROI and eROI adjacent pairs. Extravascular transport was monitored further away from the vasculature by the creation of contiguous eROIs extending at perpendicular distances to the iROI. Therefore, for the evaluation of extravasation from a particular blood vessel segment (defined by an iROI) free of fluorescence from other sources (as discussed), iROI/ eROIs pairs were selected in regions that appeared clear of any extraneous fluorescence for the duration of each video.

Selection of ROIs is further constrained by limitations of the confocal imaging region when surfaces are curved, uneven, and/or non-level under high numerical aperture (NA) giving a very thin depth of field or optical slice. Many IVM studies use a window chamber in the animal, which sandwiches tissue between two plates keeping it flat, but this artificially constrains tumor growth. In this study, exposed subcutaneous tumor was imaged free of a window chamber, and thus, certain regions present a curved surface. In this case, the optical section is smaller than the full FOV because tumor tissue extending too far above the imaging plane toward the objective lens prevents imaging in that region, while the tumor surface 
on the opposite side falls away from the imaging plane. ${ }^{43}$ For a curved tumor surface, fluorescence in the optical slice exists within a curved region. If ROIs stray out of this region, reduction in fluorescence intensity will contain artifact. ROIs in this study were selected to minimize such artifacts.

\section{Local ROI analysis and avoidance of movement artifacts}

To study the dynamics of extravasation, localized ROIs were selected to avoid imaging artifacts and distribution across connected tumor regions, both within blood vessels at the scale of vessel dimensions, and extravascularly with incrementally increasing distance perpendicular to specific $\mathrm{C}_{60}$-serPFpermeable vessels. The spatially localized data sets obtained from these ROIs permit approximation of the type of transport occurring in various biological compartments. Extensive heterogeneity of tumor tissue across animals, tumors, and even within the same tumor can make valid comparisons between experiment and control samples challenging to obtain. This method of selection of local ROIs enables the analysis of regions over time relative to earlier time points and, thus, avoids the problems of incorrectly comparing NP kinetics across morphologically variable structures that may interact differently with the NPs. Furthermore, we are free to choose regions that will avoid or minimize optical artifacts, making the most of the strengths of confocal microscopy in localizing regions in three-dimensional space while minimizing its limitations, such as by avoiding regions in the FOV where light cannot penetrate due to an uneven tissue surface. ${ }^{43}$

Because local ROIs are static, a small amount of error occurred where blood vessels briefly moved in and out of the focal plane or translated slightly in the $X$ and $Y$ directions. This was minor overall for the ROIs selected, and eROIs were trimmed to avoid contamination of eROI by extraneous blood vessel fluorescence in cases where blood vessels not directly involved in the measurements moved into eROIs. Vessel diameter was determined based on blood vessel perfusion with FITC-dextran, assuming a negligible amount extravasated within the timeframe imaged $(2 \mathrm{~min})$.

\section{Global ROI analysis}

A global thresholding algorithm was developed to segment $\mathrm{C}_{60}$-serPF fluorescence in the IVM images into two regions, the intravascular region, defined by fluorescence in the FITC channel, and the extravascular region, the inverse of the former region, to quantify NP extravasation. NIS-Elements software was used to produce the mask of the vasculature, which was then superimposed on a second mask created from $\mathrm{C}_{60}$-serPF fluorescence in the Cy5 channel of the same image FOV. The threshold of $\mathrm{C}_{60}$-serPF fluorescence was calculated from a single frame in the image sequence shortly after the appearance of $\mathrm{C}_{60}$ ser-PF fluorescence in the image FOV. This threshold was then applied to $\mathrm{C}_{60}$-serPF fluorescence in each frame of the entire image sequence to create the $\mathrm{C}_{60}$-serPF mask. We had found that voxels of intensity values present at high frequency throughout an image tended to be noise or artifact, as could be demonstrated by visual inspection. Therefore, threshold selection criteria for $\mathrm{C}_{60}$-serPF were based on finding a minimum intensity value with a low enough frequency (low number of voxels) in the image to contain useful information.

Having defined masks representing both the intravascular and extravascular space, as well as the region occupied by $\mathrm{C}_{60}{ }^{-}$ serPF, these masks were applied to measure $\mathrm{C}_{60}$-serPF mean intensities in the intravascular and extravascular compartments (the global iROI and the global eROI) respectively over the course of an image sequence. To establish a baseline at zero for each of the graphs produced, the minimum intensity across the entire time interval was taken as background and subtracted from each of the points in both ROIs for all images in the series.

\section{Results \\ Physicochemical characterization of $\mathrm{C}_{60}$-ser}

As a basis for understanding the aqueous-phase behavior of $\mathrm{C}_{60}$-ser, its physicochemical properties were characterized in terms of aggregate morphology (by SEM), aggregate size distribution (by DLS), and zeta potential, a measure of surface charge. With a solubility in water exceeding $250 \mathrm{mg} / \mathrm{mL}, \mathrm{C}_{60}{ }^{-}$ ser is an amphipathic molecule composed of a hydrophobic [60]fullerene core derivatized with six hydrophilic malonodiserinolamide chains bearing a total of 24 hyroxyls (Figure 1A). Due to this special amphipathic geometry, $\mathrm{C}_{60}$-ser aggregates in aqueous solutions in shell-like arrangements. Figure 1 shows aggregates of $\mathrm{C}_{60}$-ser in SEM, where lyophilization (flash freezing and drying) of $\mathrm{C}_{60}$-ser has preserved its aqueous-phase aggregate morphology. Hollow shells ranging from $\sim 100 \mathrm{~nm}$ to several microns in diameter are shown in Figure $1 \mathrm{~B}$ at $15,000 \times$ magnification. Arrows indicate transparency and breakage of the shells (due to rapid escape of water under high vacuum conditions during lyophilization), revealing that shell thickness is likely on the order of a monolayer of $\mathrm{C}_{60}$-ser molecules. Figure $1 \mathrm{C}$ and D shows SEM images at 1,000,000× magnification elucidating grains in the structure of the shells believed to be single $\mathrm{C}_{60}$-ser molecules with sizes corresponding closely to its hydrodynamic diameter of $\sim 3 \mathrm{~nm}$.

DLS measurements performed on a Malvern Zetasizer Nano illustrate the dynamics of particle size distributions of $\mathrm{C}_{60}$-ser aggregates and $\mathrm{C}_{60}$-ser single molecules dissolved in 

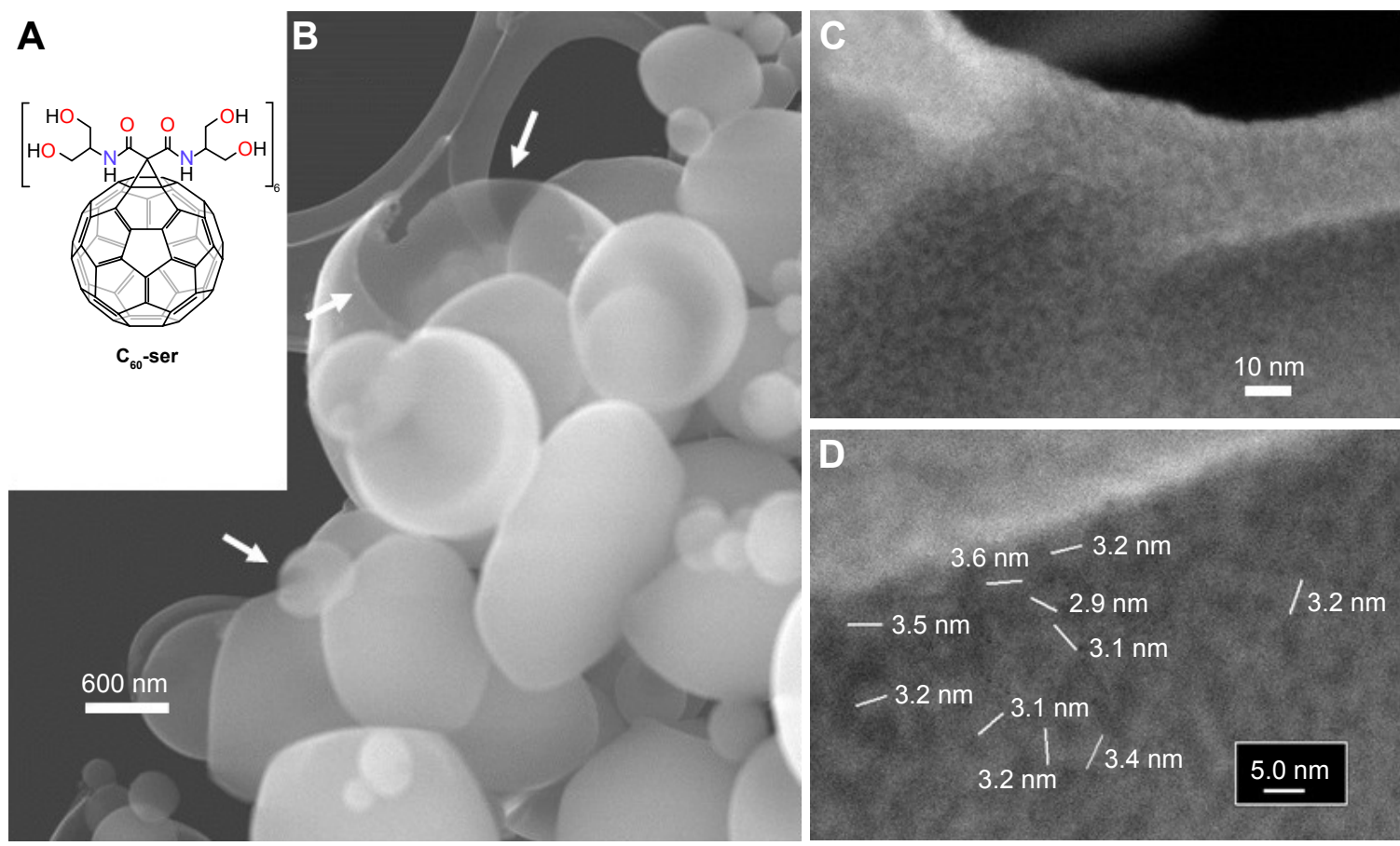

Figure I Malonodiserinolamide-derivatized [60]fullerene $\left(\mathrm{C}_{60}\right.$-ser) molecular structure and aggregation morphology.

Notes: Molecular structure of $\mathrm{C}_{60}$-ser (A). SEM of $\mathrm{C}_{60}$-ser aggregates lyophilized from a solution of $10 \mathrm{mg} / \mathrm{mL}$ in DI water using secondary electron detector at (B) I5,000X and (C) I,000,000 $\times$ magnifications acquired at $30 \mathrm{kV}$. Arrows in (B) point out transparency of aggregate shells and/or breaks in shells. Individual grains ( $\mathrm{C}_{60}$-ser molecules) within a single aggregate shell in (C) are shown in zoom view (D) with representative measurements of $\mathrm{C}_{60}$-ser molecules, $3.3 \pm 0.39 \mathrm{~nm}$, mean diameter \pm SD.

Abbreviations: DI, deionized; SEM, scanning electron micrographs.

either DIW or phosphate-buffered saline (PBS) at pH 7.2. In Figure 2, particle size distribution is plotted against time beginning immediately after dissolution of $\mathrm{C}_{60}$-ser powder at various concentrations. Based on the limitations of DLS to accurately size particles $<20 \mathrm{~nm}$ in diameter, ${ }^{44}$ particles in this size range are taken to be single $\mathrm{C}_{60}$-ser molecules and particles above this size range are taken to be aggregates. In DIW at the concentrations of $0.5,1$, and $10 \mathrm{mg} / \mathrm{mL}$ (top row of Figure 2), aggregates had already formed within the earliest possible reading at $\sim 2.5 \mathrm{~min}$ after dissolution from dry powder and these aggregates stayed within a constant size range for the duration of the experiment. The median aggregate size was observed to be $\sim 100-200 \mathrm{~nm}$. At 0.5 and $1.0 \mathrm{mg} / \mathrm{mL}$, a small percentage of single $\mathrm{C}_{60}$-ser molecules coexisted with aggregates, while at $10 \mathrm{mg} / \mathrm{mL}$, no single molecules were detected. A small percentage of larger $\mathrm{C}_{60}$-ser aggregates, several microns in size, are also observed in the DLS graphs.

In contrast, in PBS (bottom row of Figure 2), aggregate behavior differed somewhat from that in deionize water. The graphs show that after the first several hours, $\mathrm{C}_{60}$-ser aggregates increased in size by several hundred nanometers and subsequently stabilized, with a narrowing of the size range and reaching dynamic equilibrium with a small percentage of single $\mathrm{C}_{60}$-ser molecules ( 1 and $10 \mathrm{mg} / \mathrm{mL}$; Figure 2) or no free $\mathrm{C}_{60}$-ser molecules (at $0.2 \mathrm{mg} / \mathrm{mL}$ in PBS).
Figure 3 shows dynamics of zeta potential of $\mathrm{C}_{60}$-ser over time at concentrations $0.05,1$, and $10 \mathrm{mg} / \mathrm{mL}$ after dissolution in DIW. Initially, at all concentrations of $\mathrm{C}_{60}{ }^{-}$ ser, zeta potential distributions were centered near $-50 \mathrm{mV}$. At lower concentrations $(0.05$ and $1 \mathrm{mg} / \mathrm{mL})$, the distribution of zeta potentials was initially unstable and then stabilized over several hours. At the very low concentration of $0.05 \mathrm{mg} / \mathrm{mL}$ (near the lower limit of Zetasizer readings), the zeta potential drifted toward neutral and eventually stabilized near $\sim-25 \mathrm{mV}$. At $1 \mathrm{mg} / \mathrm{mL}$, the zeta potential drifted more subtly toward neutral, stabilizing $\sim-30 \mathrm{mV}$, while at the high concentration of $10 \mathrm{mg} / \mathrm{mL}$, the zeta potential stayed relatively constant between approximately -40 and $-60 \mathrm{mV}$. Zeta potential can be taken as a measure of surface charge, with these negative readings suggesting that the $\mathrm{C}_{60}$-ser aggregates carry a negative surface charge in DIW.

\section{Intratumoral vascular, extravasation, and extravascular transport kinetics of $\mathrm{C}_{60}$-serPF in vivo}

In Figure 4, a time series of confocal images shows vascular delivery and extravasation of $\mathrm{C}_{60}$-serPF to tumor microvasculature of mouse $\# 1$ at $10 \mathrm{~s}$ intervals, with the first frame $10 \mathrm{~s}$ after coinjection of FITC-conjugated dextran (FITC-dextran) and $\mathrm{C}_{60}$-serPF. Fluorescence images from 


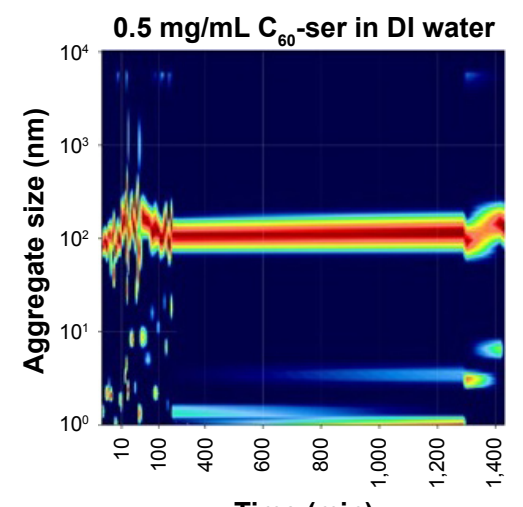

Time (min)

$0.2 \mathrm{mg} / \mathrm{mL} \mathrm{C}_{60}$-ser in PBS (pH 7.2)

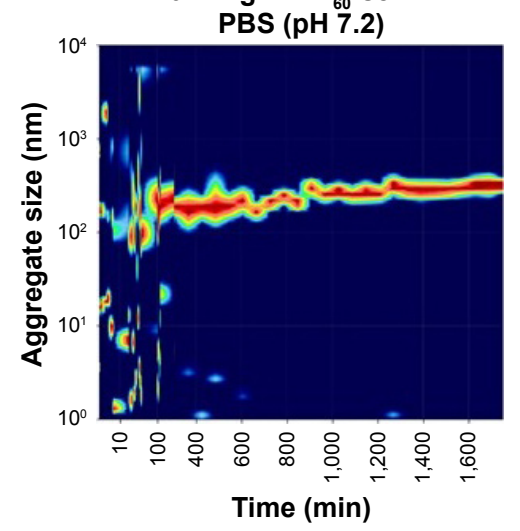

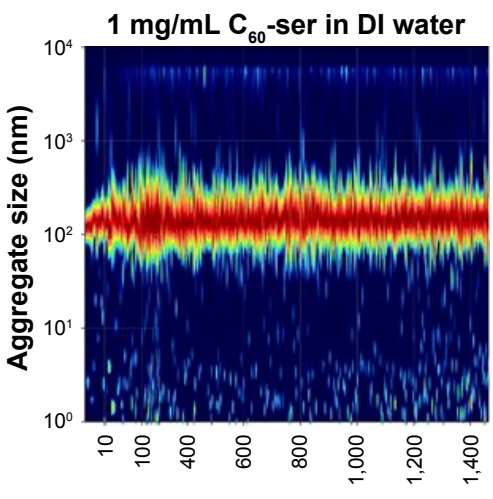

Time (min)

$1 \mathrm{mg} / \mathrm{mL} \mathrm{C} \mathrm{C}_{60}$-ser in

PBS (pH 7.2)

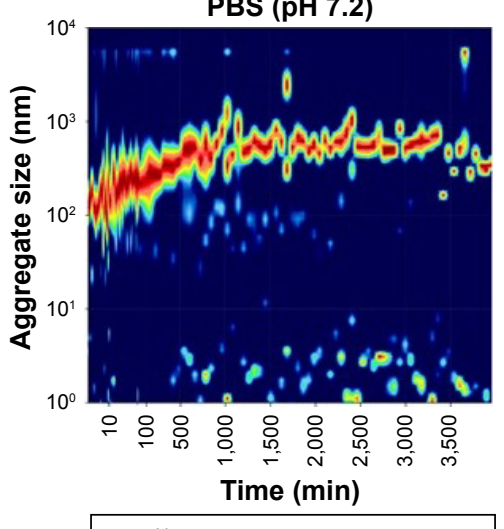

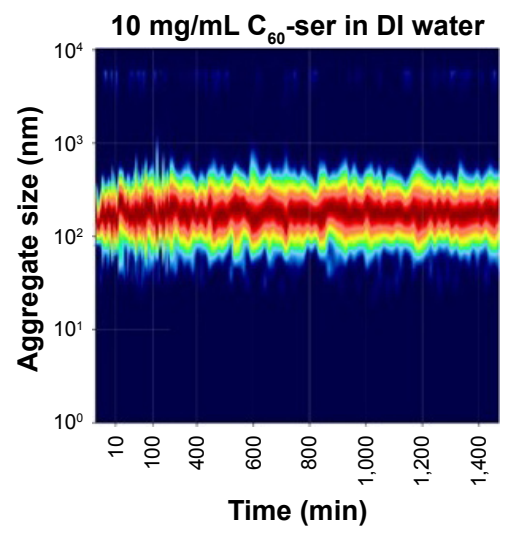

$10 \mathrm{mg} / \mathrm{mL} \mathrm{C}_{60}$-ser in PBS (pH 7.2)

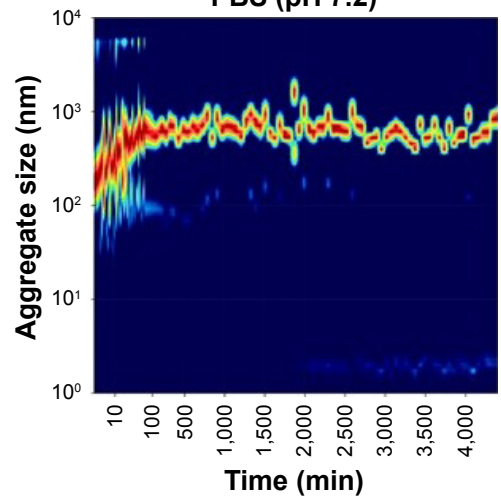

บ $0 \%: 4 \square 8=16 \square 32 \square 64$

Figure 2 Dynamics of $C_{60}$-ser size distribution across various $C_{60}$-ser concentrations.

Notes: Time course of particle size distribution (hydrodynamic particle diameters) by DLS after the dissolution of $\mathrm{C}_{60}$-ser lyophilized powder at concentrations 0.5 , I, and $10 \mathrm{mg} / \mathrm{mL}$ in deionized water (top row) and $0.2,1$, and $10 \mathrm{mg} / \mathrm{mL}$ in PBS, pH 7.2 (bottom row). Heat maps denote the percentage of total particles at each hydrodynamic diameter.

Abbreviations: DLS, dynamic light scattering; PBS, phosphate-buffered saline.

each channel are shown together in false color (top series) and separately in FITC (FITC-dextran, middle series) and Cy5 channels (bottom series). At $10 \mathrm{~s}$ postinjection, both particles are imaged entering an arteriole (top left of all images) followed by filling of the capillary bed 30-50 s postinjection. From 30 to $50 \mathrm{~s}$ postinjection, $\mathrm{C}_{60}-\mathrm{serPF}$ extravasates and diffuses through the interstitial region. This extravasation is evident in the top panel series, where
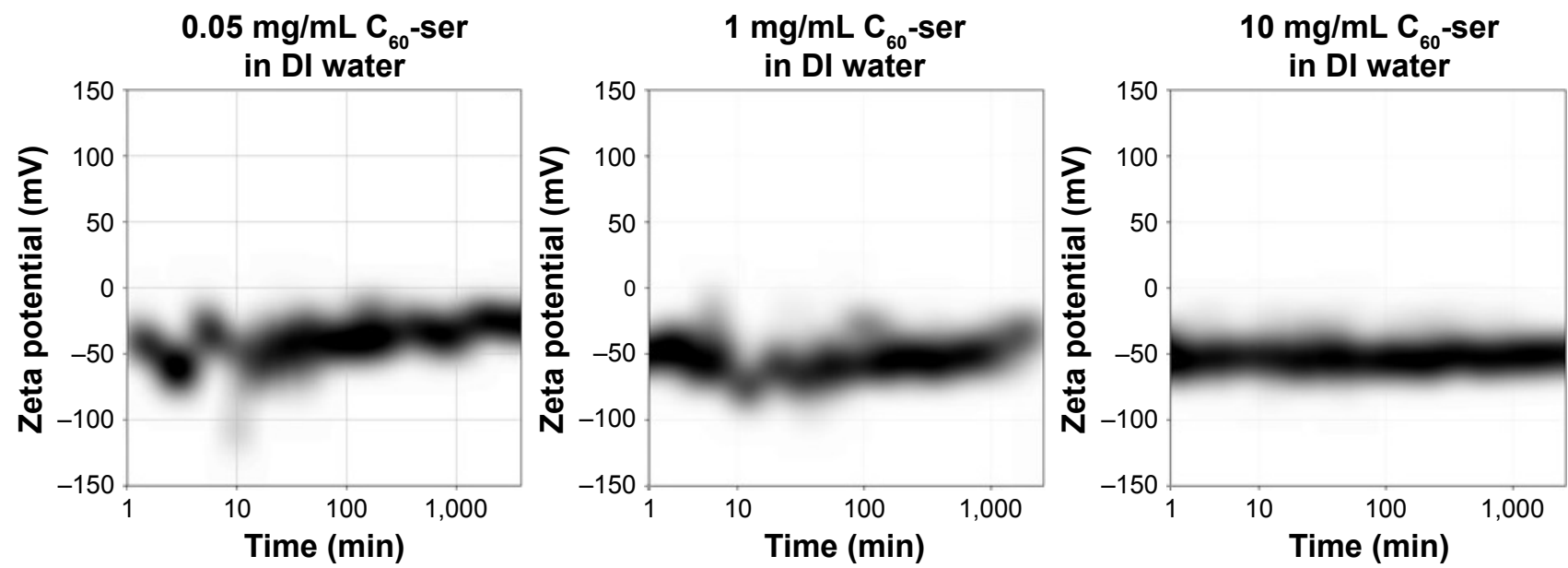

Figure 3 Dynamics of $\mathrm{C}_{60}$-ser zeta potential across various concentrations.

Notes: Time course after dissolution of $\mathrm{C}_{60}$-ser at concentrations $0.05,1$, and $10 \mathrm{mg} / \mathrm{mL}$ in deionized water. Zeta potential measurements were more stable at $10 \mathrm{mg} / \mathrm{mL}$ than at 0.05 and $\mathrm{I} \mathrm{mg} / \mathrm{mL}$. At lower concentrations $(0.05$ and $\mathrm{I} \mathrm{mg} / \mathrm{mL}), \mathrm{C}_{60}$-ser zeta potential tended toward neutral over time. 
A
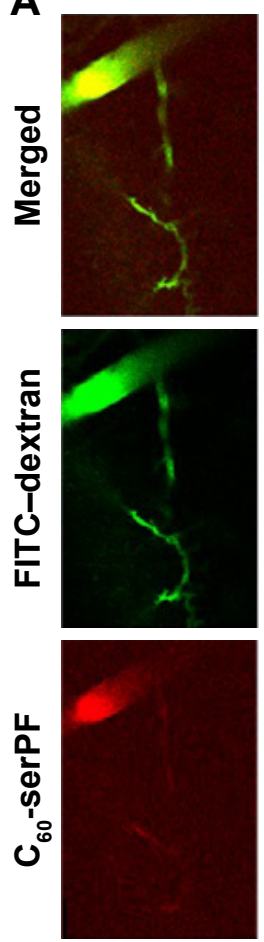

$11 \mathrm{~s}$
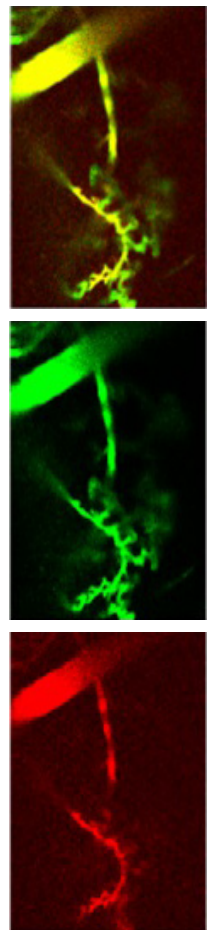

$21 \mathrm{~s}$
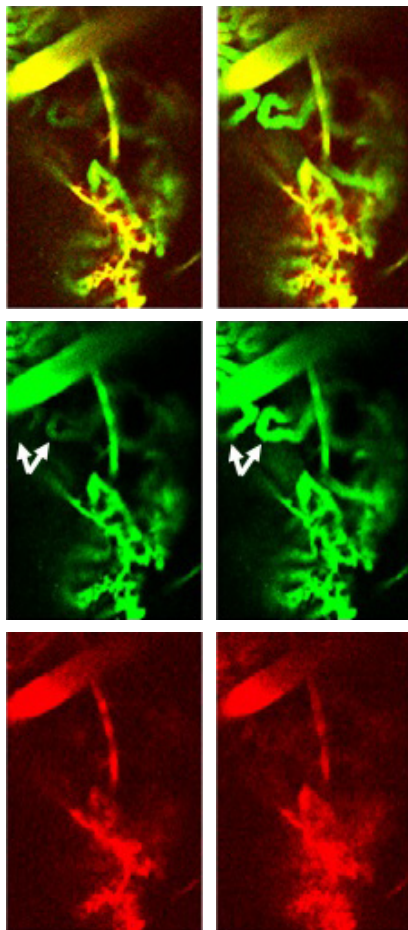

$31 \mathrm{~s}$

$42 \mathrm{~s}$
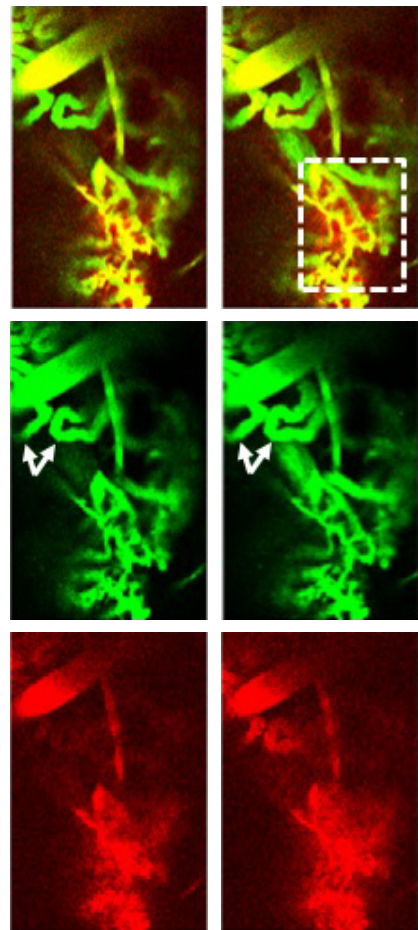

B
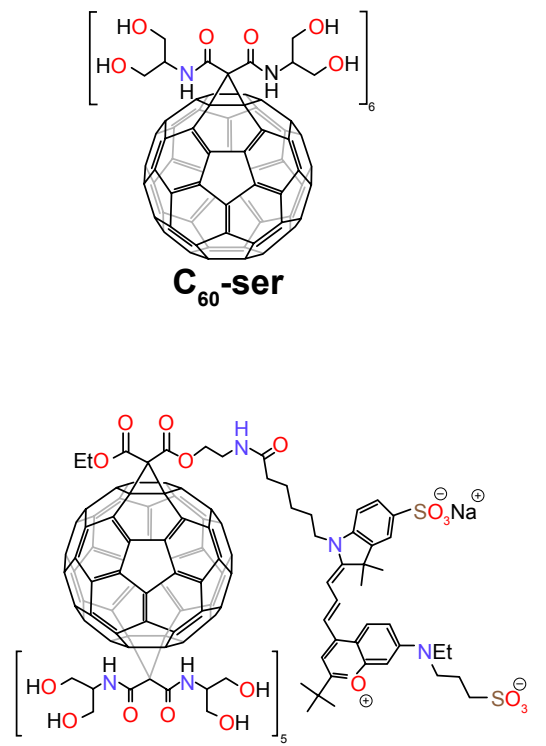

$\mathrm{C}_{60}$-serPF

Figure 4 IVM image sequence of simultaneous systemic injection of $\mathrm{C}_{60}$-serPF and vascular tracer in tumor microvasculature of tumor-bearing mouse \#I.

Notes: (A) Images taken at $\sim 0.5$ fps at I6× magnification using a Galvano scanner show FITC-dextran (vascular tracer) and $\mathrm{C}_{60}$-serPF (NP) delivery to tumor microvasculature upon retro-orbital coinjection of these agents in a mouse. Middle and bottom rows show false-color FITC (FITC-dextran; green) and Cy5 (C 60 -serPF; red) channels, respectively, while top row shows both channels merged. The approximate time postinjection of agents is shown at the bottom of each column. Arrows in the middle row show appearance of blood vessels with a time lag in NP and tracer perfusion. The inset in the last frame in the top row shows the region of analysis in Figure $5 \mathrm{~A}$ and $\mathrm{B}$. (B) Molecular structures of injected NPs. Video SI corresponds to the image sequence, images, and graphs in this figure and in Figure 5A and B.

Abbreviations: IVM, intravital microscopy; NPs, nanoparticles.

false color red $\left(\mathrm{C}_{60}\right.$-serPF fluorescence) bleeds out through the false color yellow capillaries (colocalization of red and green dyes). Of interest, at $\sim 30$ s postinjection, additional vessels appear in the video (such as those directly below the large diameter blood vessel at the upper left of the images), with a greater relative fluorescence intensity of FITC to Cy5 compared to that of vessels appearing in the first pass at $\sim 10 \mathrm{~s}$ postinjection. Both the delay in perfusion of certain tumor blood vessels and differences in relative fluorescence intensities of FITC-dextran and $\mathrm{C}_{60}$-serPF in these blood vessels are analyzed in the following sections (Figure 8). Imaging proceeded for $2 \mathrm{~min}$ postinjection.

To quantify extravasation and transport kinetics of $\mathrm{C}_{60}$-serPF in the tumor interstitium, mean fluorescence intensity (assumed proportional to dye concentration) was measured in ROIs within microvasculature (iROIs) and immediate surrounding extravascular regions (eROIs) in the focal plane (Figure 5). In Figure 5A, ROI-1 is an iROI in an $11 \mu \mathrm{m}$ diameter capillary and ROIs $2-4$ are similarly sized eROIs at successively increasing perpendicular distances from the capillary wall. The graph of fluorescence intensity of $\mathrm{C}_{60}$-serPF in the Cy5 channel versus time (bottom of Figure $5 \mathrm{~B}$ ) shows that shortly after bolus injection $(t=0)$, the fluorescence intensity of ROI- 1 in mouse \#1 underwent a pulse increase from background level, while eROIs with increasing distance from the vasculature underwent successively gradual increases in intensity. Due to preset laser power and gain settings, saturation of the fluorescence signal occurred in both Cy5 and FITC channels intravascularly in certain regions, although not in the eROIs.

Intravascular signal saturation occurred in ROI-1 (Figure 5A of mouse \#1 and RO1-1 of Figure 5C in the subcutaneous microvasculature of a normal mouse). Imaging in the FITC channel across the same ROIs as in the Cy5 channel serves as an important internal control for $\mathrm{C}_{60}-\mathrm{serPF}$ extravasation, as there is a comparatively negligible extravasation of FITC-dextran. While confocal microscopy has the distinct advantage over epifluorescence microscopy of fluorescence imaging in a single well-defined plane, fluorescence from adjacent parallel planes is excluded only by a pinhole at the detector and some light leakage (visible in 
A
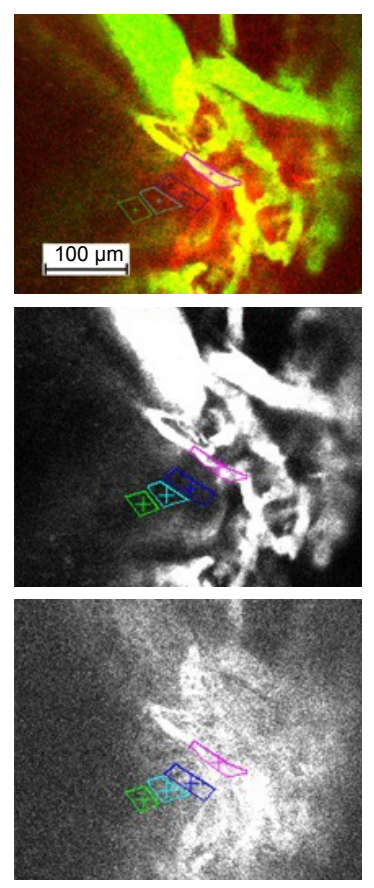

B
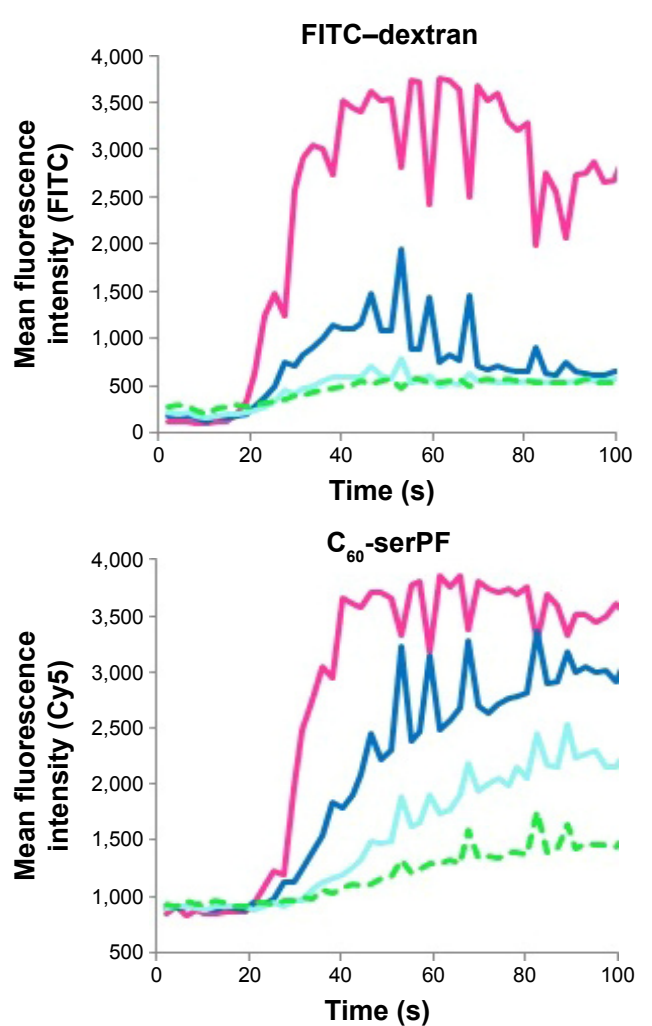

C
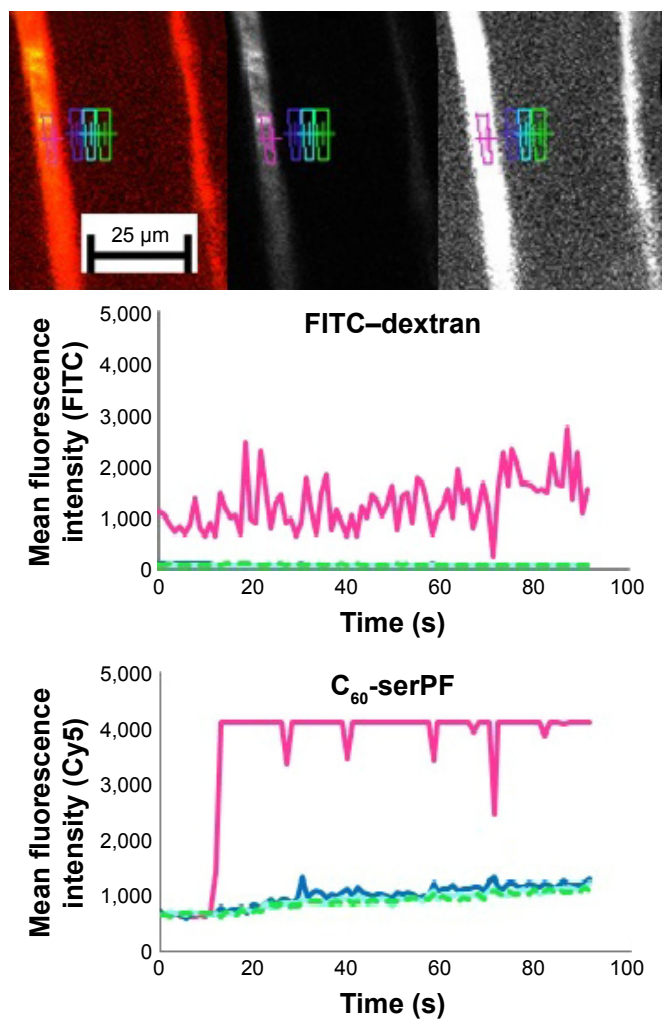

$\longrightarrow$ ROI-1 ROI-2 ROI-3 - - ROI-4

Figure 5 IVM images of delivery of $\mathrm{C}_{60}$-serPF to tumor microvasculature of mouse \#I compared to delivery to normal mouse with corresponding dynamic changes in fluorescence intensity across local ROls.

Notes: (A) $\mathrm{C}_{60}$-serPF in Cy5 channel, FITC-dextran in FITC channel, and both agents merged in dual channel confocal microscopy images at I6× magnification using a Galvano scanner (bottom to top) in tumor microvasculature and interstitium at $74 \mathrm{~s}$ post coinjection of NPs. ROI-I in a segment of tumor microvasculature (iROI) and ROls 2-4 at successively increasing distances into tumor interstitium perpendicular to capillary wall (eROls) are superposed on images. (B) Mean fluorescence intensity versus time in Cy5 (top) and FITC (bottom) channels in ROls I-4 are color coded corresponding to ROls shown in (A); time of injection at $t=0$ s. (C) Cy5, FITC, and dual channel images (top, right to left) at $78 \mathrm{~s}$ postinjection of $\mathrm{C}_{60}$-serPF (where FITC-dextran had been injected $\sim 20$ min earlier) in microvasculature of a normal mouse at $16 \times$ magnification using a Galvano scanner; corresponding graphs of fluorescence versus time of $\mathrm{C}_{60}$-serPF and FITC-dextran (bottom and middle) delivery to microvasculature. Video SI corresponds to the image sequence, images, and graphs in Figure 4 and in (A) and (B). Video S2 corresponds to images and graphs in (C).

Abbreviations: eROls, extravascular ROls; iROls, intravascular ROIs; IVM, intravital microscopy; ROls, regions of interest.

the imaging plane) can occur. In this figure (graph at top of Figure 5B and corresponding to the image in the middle of Figure 5A), a similar pulse of FITC-dextran fluorescence intensity is observed in ROI- 1 in the capillary vessel, but the dynamics of fluorescence intensity in eROIs $2-4$ do not follow the same smooth pattern as that of $\mathrm{C}_{60}$-serPF in the Cy5 channel ROIs. A moderate amount of fluorescence in the FITC channel within these ROIs may be due to the perfusion of capillaries near the imaging plane as well as some small amount of extravasation. Since the capillary bed of a tumor is extremely tortuous and dense, it is often impossible to completely avoid fluorescence from nearby out-of-plane vessels. However, comparison of changes in fluorescence between FITC and Cy5 channels in extravascular regions suggests that there is likely only a minimal contribution of out-of-plane fluorescence to the fluorescence intensity of $\mathrm{C}_{60}$-ser extravasation.
Tumor vascular permeability was also assessed in comparison to $\mathrm{C}_{60}$-serPF permeability in normal vasculature. Figure 5C shows images and corresponding graphs of changes in fluorescence intensity in corresponding iROIs and eROIs in the subcutaneous vasculature and tissue of a normal mouse. Normal vasculature is far less dense and more organized than tumor vasculature, and therefore, it is not difficult to select ROIs that avoid the overlap of fluorescence from the proximity of other vessels either within or across focal planes. Upon injection of $\mathrm{C}_{60}$-serPF after preinjection of FITC-dextran, minimal fluorescence increase is observed in the Cy5 channel compared with that in previous figures, indicating that substantially less extravasation of $\mathrm{C}_{60}$-serPF occurs from normal vasculature.

To avoid intravascular signal saturation so that intravascular dynamics of $\mathrm{C}_{60}$-ser delivery could be observed, 
in a second experiment (Figure 6, a different animal), high voltage (HV) gain and laser power were reduced to avoid saturation upon $\mathrm{C}_{60}$-serPF bolus injection. In ROI- 1 in Figure 6 where intravascular fluorescence intensity did not saturate, a decrease in Cy5 channel fluorescence begins immediately after the bolus pulse delivery of $\mathrm{C}_{60}$-serPF to blood vessels in mouse \#2. Observation of these kinetics was likely blocked by signal saturation in the previous experiment. With fluorescence intensity in adjacent extravascular regions increasing with a similar trend to those in the previous experiment, fluorescence intensity of $\mathrm{C}_{60}$-serPF across iROIs and eROIs nearly equalizes after $\sim 1 \mathrm{~min}$. In this run, in the FITC channel for the same ROIs, differences from Cy5 channel kinetics were even more salient. After bolus pulse increase in the iROI (ROI-1), FITC-dextran fluorescence intensity did not dissipate over the time period sampled.
There was also negligible fluorescence intensity increase above background in FITC channel eROIs and less than in the previous run.

\section{Global image ROI analysis}

A global ROI analysis across the entire FOV of all images in the imaging series of the two tumor-bearing mice and one normal mouse was conducted to validate the data acquired in the local ROI analysis, extending the analysis to a substantially larger imaged region (Figure 7). Only the kinetics of the $\mathrm{C}_{60}$-serPF was tracked during the global ROI analysis as the local analysis sufficiently displayed the reliability of FITC-dextran as a vascular tracer. The measurements acquired using the NIS-Elements software were used to plot mean fluorescence intensity versus time for the global ROIs for each of the three image sets. Mean fluorescence intensity
A

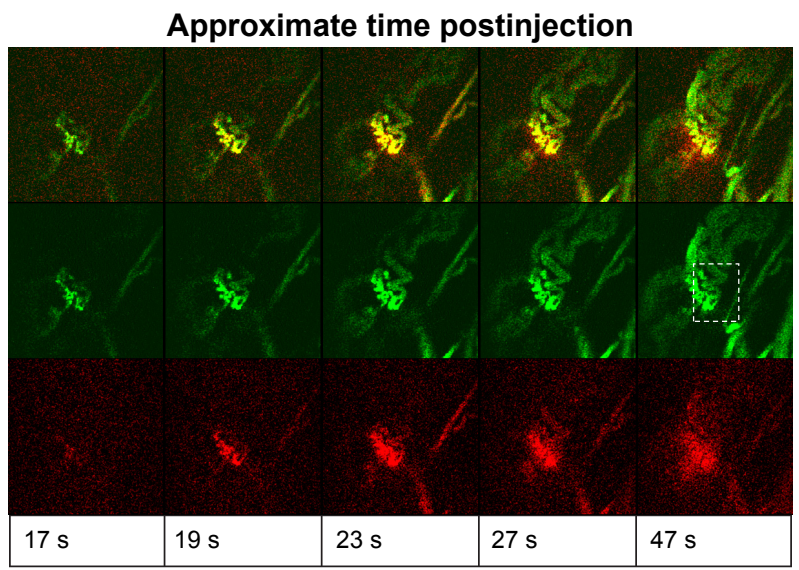

B

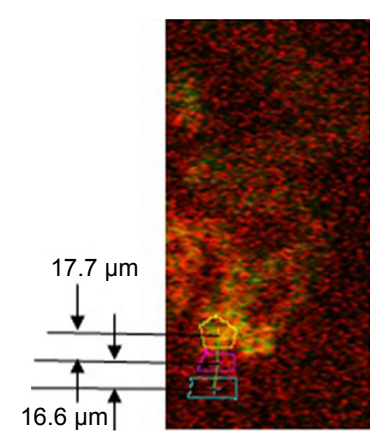

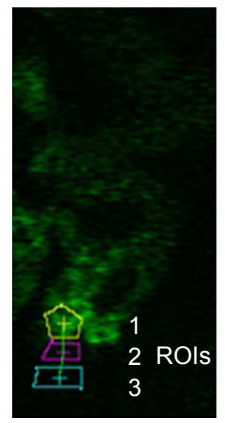

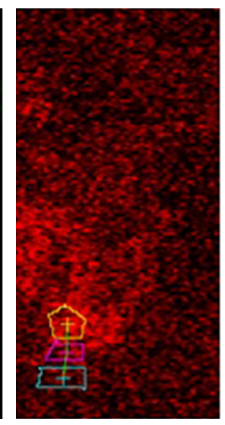

C
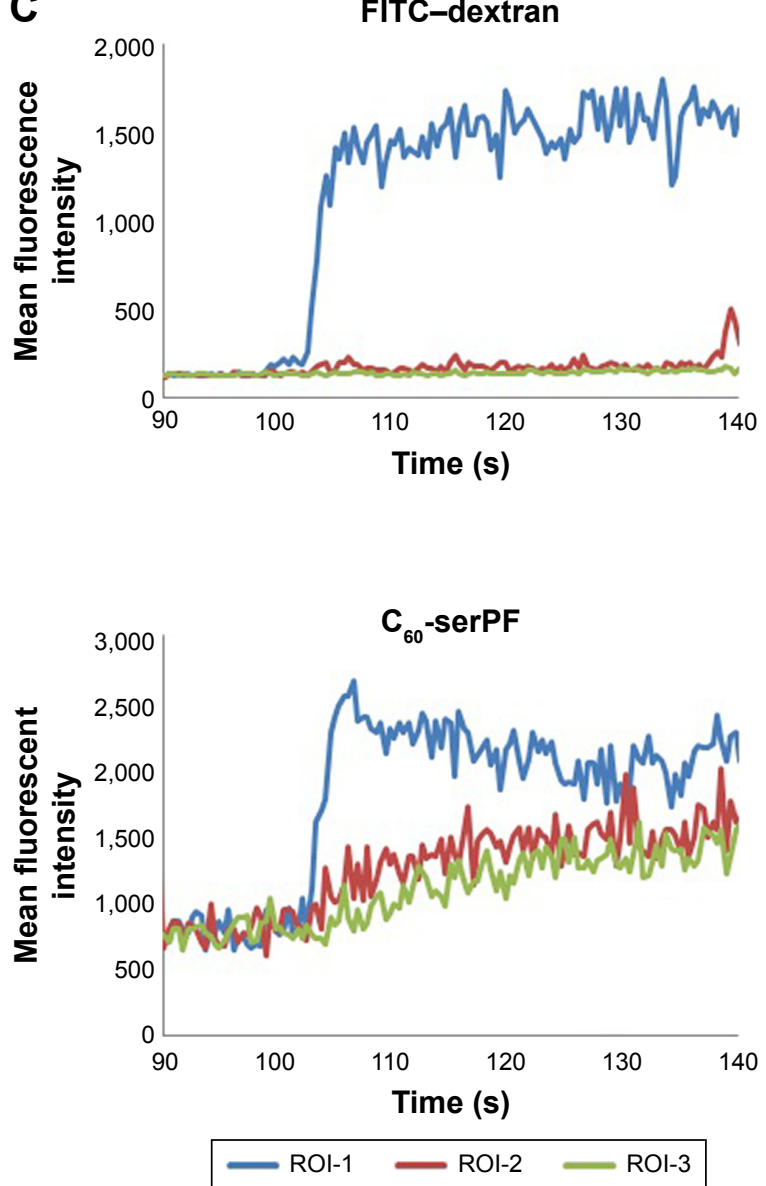

Figure 6 IVM image series and fluorescence quantification in local ROls of $\mathrm{C}_{60}$-serPF delivery to tumor microvasculature of mouse $\# 2$ where intravascular ROI fluorescence intensity did not saturate.

Notes: (A) Sequential IVM video images of $\mathrm{C}_{60}$-serPF and FITC-dextran coinjection in channels Cy5, FITC, and merged (bottom to top rows respectively) at $\sim 2.5$ fps at select time points postinjection at $16 \times$ magnification using a Resonant scanner. Brightness and contrast adjusted for clarity (refer Figure S2 for unadjusted video images). (B) Zoom view (corresponding approximately to white dotted box in last frame of middle row in [A]) showing location of intravascular (I) and extravascular ( 2 and 3 ) ROls spaced $\sim 17 \mu \mathrm{m}$ apart; brightness and contrast not adjusted. (C) Graphs of mean fluorescence intensity of corresponding ROls versus time in channels FITC (top) and Cy5 (bottom). Video S3 corresponds to the image sequence, images, and graphs in this figure, where brightness and contrast have been adjusted to reduce noise for clarity. Abbreviations: IVM, intravital microscopy; ROls, regions of interest. 

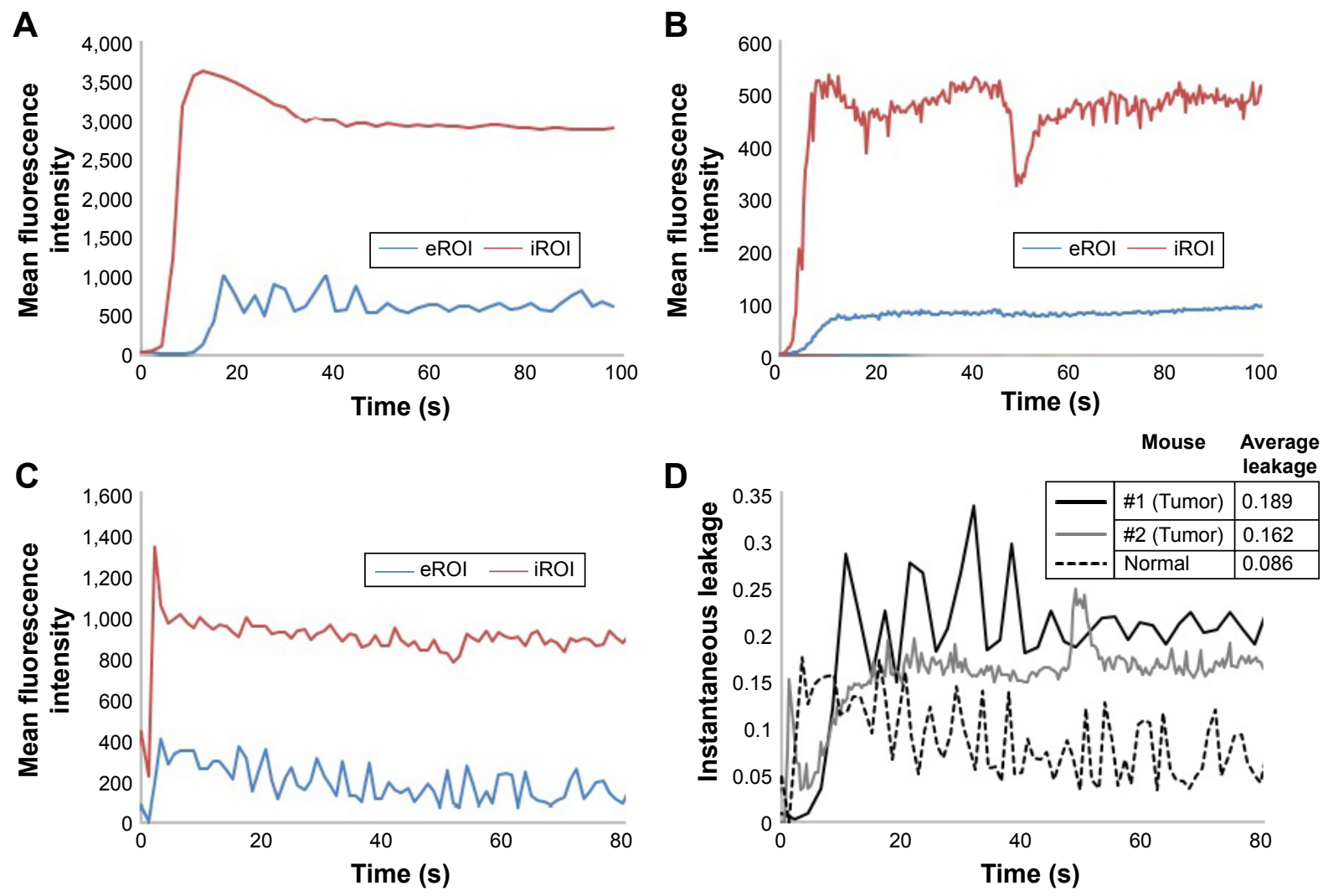

Figure 7 Global ROI analysis across entire field of view of image series.

Notes: Comparison of tumor to normal mouse vasculature. Mean fluorescence intensity of $\mathrm{C}_{60}$-serPF within global iROI and eROI for tumor-bearing mice (\#I and \#2, $\mathbf{A}$ and $\mathbf{B}$ ) and normal mouse (C) versus time, applied to same videos as for local ROls. (D) Instantaneous leakage of $\mathrm{C}_{60}{ }^{- \text {serPF }}$ versus time for the three graphs in (A-C) as well as the average instantaneous leakage calculated from the graph in (D). Background fluorescence was subtracted from all image series.

Abbreviations: eROI, extravascular ROI; iROI, intravascular ROI; ROI, region of interest.

was selected as it is proportional to the concentration of $\mathrm{C}_{60}$ ser-PF within the z-slice of the FOV.

For mouse \#1, the first $\sim 10 \mathrm{~s}$ recorded in the global ROIs displayed a rapid rise in the amount of $\mathrm{C}_{60}$-serPF present within the vasculature until reaching a maximum mean fluorescence intensity following $12.8 \mathrm{~s}$ of imaging. Mean fluorescence intensity of the global iROI then decayed steadily until reaching a steady state following $\sim 40$ s of imaging. A notable increase in the mean fluorescence intensity of $\mathrm{C}_{60}{ }^{-}$ serPF in the extravascular region lagged behind that of the intravascular $\mathrm{C}_{60}$-serPF, following $10.6 \mathrm{~s}$ of imaging before oscillating about a maximum value and eventually converging upon a steady state following $55.3 \mathrm{~s}$ of imaging. The sharpest increase in the mean fluorescence intensity within the extravascular region corresponds to the decline in mean fluorescence intensity in the intravascular region.

Mouse \#2 displayed similar initial dynamics with a rapid increase in the mean fluorescence intensity of the intravascular $\mathrm{C}_{60}$-serPF in the first $10 \mathrm{~s}$ of imaging reaching a steady state following $\sim 60 \mathrm{~s}$ of imaging. Similar to mouse \#1, the increase in the mean fluorescence intensity of extravascular $\mathrm{C}_{60}$-serPF lags behind that of intravascular $\mathrm{C}_{60}$-serPF.

For the normal mouse, there was a sudden spike in the mean fluorescence intensity for $\mathrm{C}_{60}$-serPF in both the intravascular and extravascular regions followed by a decline in both. Changes in the mean fluorescence intensity in the extravascular region appear to have paralleled those within the vasculature. Most notably, there was not a lag time prior to the quick increase in extravascular $\mathrm{C}_{60}$-serPF mean fluorescence intensity.

The dimensionless quantity "instantaneous leakage" was defined to provide an instant-to-instant comparison over time of the amount of the NP contained in each global ROI. As mean fluorescence intensity is proportional to NP concentration, instantaneous leakage provides a sample of the local biodistribution of extravasated NP relative to the amount in the vasculature at a given time within the FOV. Instantaneous leakage, $L(t)$, was computed as the ratio of the mean intensity within the area occupied by the NP in the global eROI, $I_{e}(t)$, to the mean intensity within the area occupied by the NP in the global iROI, $I_{i}(t)$, at each instant in time in the image 
series. Background subtracted mean intensities were used. The higher the value of $L(t)$, the greater the NP fluorescence within the eROI compared to the iROI. At any value $>1$, the mean fluorescence intensity of $\mathrm{C}_{60}$-serPF in the extravascular region exceeds that of the intravascular region:

$$
L(t)=\frac{I_{e}(t)}{I_{i}(t)}
$$

Times were aligned to $t=0$ for each mouse based on the frame in which $\mathrm{C}_{60}$-serPF fluorescence first appeared, allowing for a direct comparison of the instantaneous leakage of $\mathrm{C}_{60}$-serPF for each mouse. Statistical outliers for mouse \#1 and mouse \#2 were removed from Figure 7. Furthermore, these values were not included in determining the average leakage as presented in the table within Figure 7. After the first several seconds of NP transport, the instantaneous leakage for the tumor vasculature of the tumor-bearing mice was greater than that of the normal vasculature of the normal mouse. Furthermore, as time progressed, the instantaneous leakage for mouse \#1 and mouse \#2 increased. This trend has implications for drug delivery over time in this model system, namely that $\mathrm{C}_{60}$-serPF extravasated more from tumor vasculature than from the selected type of normal vasculature. The table in Figure 7D solidifies this point, showing that the average of the instantaneous leakage across time for each mouse is substantially more in tumor compared with normal vasculature. The average leakage of $\mathrm{C}_{60}$-serPF from the vasculature of mouse \#1 was 2.2 times greater than from the vasculature of the normal mouse, while the average leakage from the vasculature of mouse \#2 was 1.9 times greater than from the normal mouse.

\section{Intravascular dynamics}

Returning to IVM imaging series of the first animal (Figures 4 and 5), additional information about drug delivery dynamics can be gleaned from changes in intravascular fluorescence where extensive extravasation is not evident. Additionally, certain blood vessels (highlighted by white arrows in Figure 4) appear in the FOV at various time points lagging behind the initial bolus delivery of $\mathrm{C}_{60}$-serPF and FITCdextran, indicating spatial and temporal heterogeneity in the delivery of these NPs to the tumor. Figure 8 follows the dynamics of select iROIs over time from Figure 4 (Video S1) where no noticeable extravasation through blood vessel walls was observed. To assess changes in fluorescence
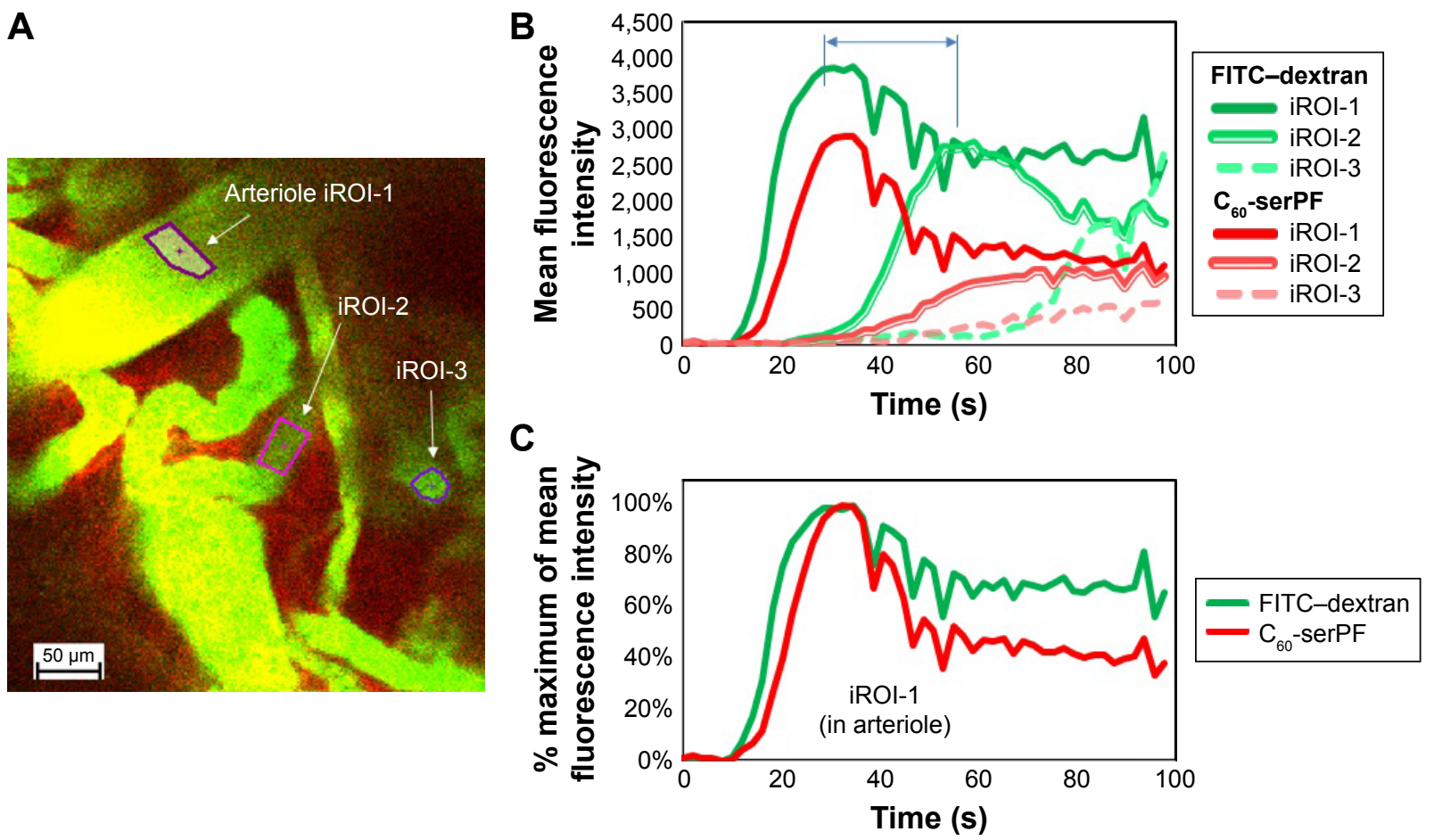

Figure 8 Fluorescence intensity of FITC-dextran and $\mathrm{C}_{60}$-serPF versus time in low-permeability tumor iROls in mouse \#I.

Notes: $(\mathbf{A})$ iROls $(\mathrm{I}-3)$ selected to demonstrate the time lag in the appearance of agents FITC-dextran and $\mathrm{C}_{60}$-serPF in successive ROls $\sim 20-30 \mathrm{~s}$ apart; image shown at $103 \mathrm{~s}$ postinjection. (B) Change in fluorescence intensities in intravascular ROls versus time where perfusion of iROI occurs 20-30 s apart. Blue arrow indicates lag between peaks of iROls I and 2. (C) Fluorescence intensity in arteriole ROI normalized as a percentage of maximum intensity reached by each agent versus time. Background fluorescence was subtracted in (B and $\mathbf{C}$ ).

Abbreviations: iROls, intravascular ROls; ROls, regions of interest. 
intensity in these ROIs, regions of fluorescence intensity saturation within the imaged FOV were avoided. The ROIs selected (Figure 8A) included one interior to an arteriole (diameter $=\sim 100 \mu \mathrm{m}$ ) and two other ROIs in tumor blood vessels to which FITC-dextran and $\mathrm{C}_{60}$-serPF were delivered in succession lagging 20-30 s. Figure 8B tracks the dynamics of mean fluorescence intensity in each of these ROIs in both FITC and Cy5 channels.

To quantify relative changes in the concentration of $\mathrm{C}_{60}{ }^{-}$ serPF compared with FITC -dextran in each of the selected ROIs in Figure 8A and $\mathrm{C}$ shows the relative fluointensities of $\mathrm{C}_{60}$-serPF and FITC -dextran as a percentage of their respective maximum intensities within the same ROI over time. By normalizing fluorescence intensities to their own maximum values within a single ROI, dyes in different channels (FTIC and Cy5) can be compared directly. Since blood flow carries new dye continuously across the FOV, photobleaching is minimized. Note that in the first (arteriole) ROI, after initial bolus increase brings both FITC-dextran and $\mathrm{C}_{60}$-serPF to their respective maximum values, by 100 s postinjection, $\mathrm{C}_{60}$-serPF dropped to $\sim 40 \%$ of its maximum value, while FITC -dextran reduced to $\sim 70 \%$ of its maximum. As shown in Figure $8 \mathrm{~B}$, $\mathrm{C}_{60}$-serPF displays different dynamics in the second and third vessels. In both cases, there is a steady rise in fluorescence, although each is diminished in intensity compared to other vessels coming into view earlier in Video S1.

\section{Discussion}

\section{Physicochemical characterization of $\mathrm{C}_{60}$-ser}

Water soluble [60]fullerene derivatives, with outer-to-inner hydrophilic-to-hydrophobic structure, exhibit differential physicochemical behavior versus amphiphilic molecules with other geometries and form larger aggregates believed to result from the strongly hydrophobic fullerene core. ${ }^{45}$ Fullerene aggregate diameters have been reported with an average hydrodynamic diameter ranging from 120 to $145 \mathrm{~nm}^{7}$ in accordance with the DLS data in our study; however, the dynamic nature of aggregate formation had not been emphasized. In addition to concentration dependence, aggregation behavior of water-soluble fullerenes has been observed to be $\mathrm{pH}^{4}$ and salt concentration ${ }^{46}$ dependent. Based on the physicochemical behavior of water-soluble fullerenes in the physiological regime, aggregation phenomena may play a role in the behavior of water-soluble fullerenes when introduced to biological systems. ${ }^{4}$

The wide range and dynamic variability in $\mathrm{C}_{60}$-ser aggregate sizes observed in our study may have implications for its intratumoral bioactivity and bioavailability. The enhanced permeation and retention (EPR) effect occurs for NPs between $\sim 10$ and $100 \mathrm{~nm}$ in diameter, where the upper bound is due to the removal of large NPs from circulation by the MPS (limiting NP access to the tumor) and the lower bound is required to hinder transport of NPs that have been delivered to the tumor from returning to circulation. ${ }^{47,48}$ The DLS data in Figure 2 show that the broad range of $\mathrm{C}_{60}$-ser sizes observed within the first hour of its dissolution in PBS at various concentrations includes some percentage within the EPR regime ( $<100 \mathrm{~nm})$, and therefore, some aggregates may undergo the EPR effect intratumorally. Dynamic equilibrium between aggregates and single $\mathrm{C}_{60}$-ser molecules may also have implications for bioavailability. For example, disaggregation of water-soluble fullerenes may contribute to the bioavailability of $\mathrm{C}_{60}$-ser and its potential drug payload at the cellular and subcellular levels and possibly offer an explanation for cellular uptake of $\mathrm{C}_{60}$-ser aggregates, such as observed previously. ${ }^{21,25}$

\section{Intratumoral extravasation and extravascular transport dynamics of $\mathrm{C}_{60}$-serPF}

While NP vascular delivery, extravasation and changes in intravascular and extravascular NP concentration have been quantified over various time scales from minutes ${ }^{31,34,39}$ to days ${ }^{35}$ using time lapse imaging, this article presents time lapse imaging with continuous quantification of NP extravasation on a second-to-second timescale in select and global ROIs, representing water-soluble fullerene derivative biotransport dynamics. At the tumor periphery, where IVM imaging has captured these transport dynamics, convective forces play a role in microvascular delivery, ${ }^{32}$ while both convection and diffusion contribute to extravascular transport. Trends in ROI fluorescence versus time observed in the quantification of IVM time lapse images suggest that convective transport dominates during initial intravascular delivery. Both molecular agents (FITC-dextran and $\mathrm{C}_{60}$-serPF in FITC and Cy5 channels, respectively) exhibit a pulse increase in fluorescence intensity occurring in local and global iROIs shortly after bolus intravenous injection of $\mathrm{C}_{60}$-serPF and FITC-dextran in both tumor-bearing and normal mice (Figures $5 \mathrm{~B}$ and $\mathrm{C}$ and $6 \mathrm{C}$ ). In contrast, in the tumor-bearing mice (Figures 5B and $6 \mathrm{C}$ ), fluorescence intensity curves for $\mathrm{C}_{60}$-serPF in the local eROIs have substantially gentler slopes that become progressively gentler with increasing perpendicular distance from the local iROI, indicating diffusive transport. ${ }^{49}$ These findings are consistent with previous assertions that due to 
high interstitial fluidic pressure in tumors, interstitial NP transport is diffusive overall. ${ }^{32}$ Global eROIs (Figure 7) do not appear to consistently elucidate these dynamics. This difference between local and global dynamics is likely due to the inclusion of nonextravasating blood vessels present in the FOV in global ROI calculations.

Variability in tumor characteristics including size, shape, and tissue density along with imaging variability due to tumor positioning under the objective lens and penetration of fluorescence through tissue to the microscope may lead to the unpredictability of optimal data collection settings including laser power and gain (experiential observations). Suboptimal imaging settings can result in the loss of information due to signal saturation at one extreme and low signal-to-noise ratio at the other. In Figure $5 \mathrm{~A}$ and $\mathrm{C}$, while saturation of intravascular $\mathrm{C}_{60}$-serPF fluorescence intensity upon bolus delivery limits information on intravascular kinetics, extravascular kinetic information was preserved, since the intensity of extravasated fluorescent $\mathrm{C}_{60}$ ser-PF fell within the dynamic range.

Given the limitations on confocal microscopy in terms of imaging unlevel surfaces and limited penetration depth, precise quantitative analysis of NP and molecular transport in the tumor microenvironment can be especially challenging due to dense and heterogeneous vasculature and ECM. The vascular tracer, FITC-dextran, therefore, serves as an important internal control in this analysis in the following respects: 1) on a short timescale, it delineates iROIs from eROIs and 2) in cases where fluorescence in eROIs occurs in the FITC channel possibly due to small amounts of FITCdextran extravasation or light leakage from perfusion of blood vessels in adjacent optical planes, its dynamic behavior was observed to be qualitatively different than that of $\mathrm{C}_{60}$-serPF. For example, in the FITC channel of tumor-bearing mice (Figures 5A and $\mathrm{B}$ and 6), relative to iROI fluorescence intensity in each respective channel, the fluorescence intensity of FITC-dextran in the eROIs in the FITC channel is far less than that of $\mathrm{C}_{60}$-serPF in the $\mathrm{Cy} 5$ channel in these experiments.

\section{Differential extravasation between tumor and normal microvasculature}

While extravasation clearly occurs in the tumor microenvironments shown in Figures $6 \mathrm{~A}$ and 8 with extravascular intensities nearly reaching that of the iROI, negligible extravasation of $\mathrm{C}_{60}$-serPF was observed in the imaged normal vasculature of the mouse with no tumor (Figure 6C) upon injection of $\mathrm{C}_{60}$-serPF at a concentration equal or greater than given to the tumor-bearing mice. While intravascular fluorescence intensity saturation of $\mathrm{C}_{60}$-serPF did occur in the imaged normal vasculature, this saturation could only have amplified eROI fluorescence intensities due to extravasation. Differential extravasation between tumor and normal microvasculature could have important implications for $\mathrm{C}_{60}$-ser as a drug delivery agent. The differential extravasation observed in this study between tumor and one type of non-tumor tissue microvasculature suggests that this methodology could help determine the extent of extravasation within tumor versus normal tissues. While further studies with additional normal tissue types are needed for this determination, the permeability of tumor microvasculature is generally higher than that of normal tissues. ${ }^{32,50}$ As NP shape, ${ }^{34}$ size, ${ }^{39}$ and both vascular and NP surface charges, ${ }^{51}$ and hydrophilicity ${ }^{36,52}$ are well-established contributors to NP extravasation, differential extravasation of $\mathrm{C}_{60}$-serPF between normal microvasculature and tumor microvasculature is likely to be highly dependent on the physicochemical characteristics of $\mathrm{C}_{60}$-serPF. Differential dosing of $\mathrm{C}_{60}$-serPF to organs and tumor has been observed previously. ${ }^{21}$

\section{Implications of global ROI analysis}

Overall, trends in the dynamics displayed in the global ROI analyses of the two tumor and one normal mouse $\mathrm{C}_{60}$-serPF delivery videos were in agreement with those of the local ROI analyses. Furthermore, within the global analyses, the IVM data for tumor-bearing mouse \#2 show a similar filling of the vessels as seen in tumor-bearing mouse \#1, accounting for the similar spike in the presence of intravascular $\mathrm{C}_{60}$-serPF, followed by a delay in the mass transfer of the NP into the extravascular region. The rapid increase in the extravasation becomes muted once the mean fluorescence intensity reaches a steady state, reducing to a slight trickle after steady state is reached. Hence, the driving force for the mass transfer of $\mathrm{C}_{60}$-serPF across the endothelium (the concentration gradient between the two ROIs) was greatest in the first few seconds of imaging.

A comparison of the individual graphs of mean fluorescence intensity versus time for mice $\# 1$ and $\# 2$ reveals that $\mathrm{C}_{60}$-serPF dynamics were governed by similar kinetics across tumor microvasculature. Kinetics is notably different however between tumor and normal microvasculature. The instantaneous and average leakage parameters serve to characterize these differences. For both tumor-bearing mice, the average leakage of the NP through tumor microvasculature was approximately twice as high as that of the normal mouse vessels. Examination of the profile of instantaneous leakage over time revealed that the amount of NP in the global eROI relative to that in the global iROI increased with time for both tumor-bearing mice \#1 and \#2. In contrast, 
the instantaneous leakage for the vasculature of the normal mouse diminished as time progressed. Based on both local and global ROI analyses, extravasation of $\mathrm{C}_{60}$-serPF more readily from the tumor microvasculature tested in this study could support its use as a drug delivery agent in terms of minimizing side effects resulting from undesirable extravasation. However, given the diversity of permeabilities of the multiple types of normal vasculature encountered in systemic NP delivery, it would be necessary to repeat this study with a number of other types of normal vasculature to confirm such a drug delivery benefit of this NP. If such an effect is confirmed, since the tumor microenvironment appears to retain $\mathrm{C}_{60}$-serPF, its attachment to anticancer drugs may preferentially target tumor over normal tissue.

\section{Time-resolved fluorescence intensity data elucidate tumor intravascular transport dynamics and clearance}

Further information about tumor intravascular NP dynamics can be gained from the analysis of nonleaking blood vessels. In iROIs where direct extravasation of NPs through vessel walls is not evident, intravascular NP dynamics can be tracked over time. In the arteriole ROI in Figure 8, pulse increases in the fluorescence of both FITC-dextran and $\mathrm{C}_{60}$-serPF are followed by monotonic decrease, in agreement with similar trends observed in other IVM studies of tumor microenvironments. ${ }^{38,39,53}$ In rough agreement with Dreher et al (2006), $\mathrm{C}_{60}$-serPF was cleared from tumor vasculature faster than the larger FITC-dextran. Upon reaching peak intensity within the arteriole $\mathrm{iROI}, \mathrm{C}_{60}$-serPF fluorescence decreased to $50 \%$ maximum within $\sim 18 \mathrm{~s}$, whereas FTIC-Dextran intensity dropped to only $\sim 70 \%$ maximum within $60 \mathrm{~s}$ (Figure 8B and C). Dreher et al (2006) circulation half-lives of 3.3 and $70 \mathrm{kDa}$ dextrans were 4 and 20 min, respectively. ${ }^{39}$ However, these times are $\sim 12$ times and $\sim 9$ times slower than the respective clearance times in our study. It is not clear from Dreher et al (2006) whether these plasma clearance rates were measured from tumor or normal vasculature, which may account for the difference in clearance rates. In our study, extravasation of $\mathrm{C}_{60}$-serPF and FITC-dextran through leaky vessels may have contributed to the faster clearance rates, although substantial FITC-dextran extravasation was not observed. Whether or not $\mathrm{C}_{60}$-serPF aggregates in vivo in the blood and in what size distribution could also be a factor if large aggregates were cleared by the MPS, although these questions remain unanswered. If the study by Dreher et al measured tumor vascular permeability as well, the departure from our study may simply reflect tumor to tumor variation.
The appearance of fluorescence in two additional iROIs in Figure $8 \mathrm{~A}$ each lag the arteriole ROI by $\sim 21$ and $\sim 30 \mathrm{~s}$, respectively. These time lags within different vessels may demonstrate temporal heterogeneity in intravenous NP delivery to the tumor. Furthermore, the longer the time past the original bolus NP delivery, the less intense the maximum fluorescence of both $\mathrm{C}_{60}$-serPF and FITC-dextran. This observation suggests that the later NP or drug is delivered via microvasculature to a region of the tumor, the lower the concentration reaching that region. An alternate explanation is that these iROIs may be in blood vessel segments that fill sequentially, such that the filling of early segments may be on the arterial side and late ones on the venous side. The lower $\mathrm{C}_{60}$-serPF concentrations observed in late filling segments may thus be those on the venous side, after extravasation of $\mathrm{C}_{60}$-serPF through tumor capillaries. However, as the systemic circulation time of mice is reported to be as fast as 7-8 times per minute ${ }^{54}$ (and approximately the same under anesthesia $^{55}$ ) it is possible that the molecular agents in the time-lagging tumor blood vessels had already undergone 2-3 rounds of circulation and partial clearance, which may support the former explanation.

Although clearance may play a role in fluorescence decrease, relative intensities in the lagging ROIs may reflect the role of extravasation and possible early evidence of EPR. In the iROI perfused second to the arteriole ROI, the intensity of FITC-dextran was $70 \%$ of FITC arteriole ROI maximum intensity, whereas the corresponding $\mathrm{C}_{60}$-serPF peak intensity dropped to $34 \%$ of the arteriole iROI value. Extravasation and EPR of $\mathrm{C}_{60}$-serPF in the tumor microenvironment may indeed account for this difference. However, since the pharmacokinetic significance of drug and NP EPR is most relevant over hours to days, further study will be necessary to determine whether an EPR effect of fullerenes is observable on those timescales.

\section{Conclusion}

In this study, we have imaged in real-time and quantified the intravascular, extravasation, and extravascular biokinetics of a water-soluble [60]fullerene derivative in the tumor microenvironment. We have further shown that $\mathrm{C}_{60}$-serPF extravasates rapidly and to a great extent in tumor vasculature, while substantially less in normal vasculature over the timescales measured. Through further analysis of $(2.87 \mathrm{kDa})$ $\mathrm{C}_{60}$-serPF intratumoral intravascular dynamics compared to a $70 \mathrm{kDa}$ FITC-dextran tracer (serving as a control), we have shown that blood clearance of $\mathrm{C}_{60}$-serPF may not be explained by renal clearance alone. New methods developed for both local microvascular region and global image analyses 
were found to be in general agreement in their respective quantification of the biokinetics of $\mathrm{C}_{60}$-serPF. These findings provide an indication of the degree of local tumor vascular permeability to NPs in this size range.

\section{Acknowledgments}

JMN acknowledges support from T32 training grant in translational biology (2T32GM088129) from the National Institute of General Medical Sciences. The authors at Rice University thank the Welch Foundation (Grant C-0627, LJW) for the support of this work. The authors would like to thank Mr Muto Atsushi of Hitachi High Technologies (Clarksburg, MD, USA) for his assistance in collecting the SEM images, as well as Dr Merlyn Pulikkathara of Baylor College of Medicine (Houston, TX, USA) for her skill in manipulation of the SEM images to provide measurements of individual fullerene NPs.

\section{Disclosure}

The authors report no conflicts of interest in this work.

\section{References}

1. Kroto HW, Heath JR, O'Brien SC, Curl RF, Smalley RE. C60: buckminsterfullerene. Nature. 1985;318:162-163.

2. Krätschmer W, Lamb LD, Fostiropoulos K, Huffman DR. Solid C60: a new form of carbon. Nature. 1990;347:354-358.

3. Wharton T, Wilson LJ. Highly-iodinated fullerene as a contrast agent for X-ray imaging. Bioorg Med Chem. 2002;10(11):3545-3554.

4. Sitharaman B, Bolskar RD, Rusakova I, Wilson LJ. Gd@C $\mathrm{C}_{60}$ $\left[\mathrm{C}(\mathrm{COOH})_{2}\right]_{10}$ and $\mathrm{Gd} @ \mathrm{C}_{60}(\mathrm{OH})_{\mathrm{x}}$ : nanoscale aggregation studies of two metallofullerene MRI contrast agents in aqueous solution. Nano Lett. 2004;4:2373-2378.

5. Rašović I. Water-soluble fullerenes for medical applications. Mater Sci Technol. 2016;44:1-18.

6. Lalwani G, Sitharaman B. Multifunctional fullerene- and metallofullerenebased nanobiomaterials. Nano Life. 2013;3:1342003.

7. Zakharian TY, Seryshev A, Sitharaman B, Gilbert BE, Knight V, Wilson LJ. A fullerene - paclitaxel chemotherapeutic: synthesis, characterization, and study of biological activity in tissue culture. $J \mathrm{Am}$ Chem Soc. 2005;127(36):12508-12509.

8. Mackeyev Y, Raoof M, Cisneros B, et al. Toward paclitaxel-[60]Fullerene immunoconjugates as a targeted prodrug against cancer. Nanosyst Phy Chem Math. 2014;5:67-75.

9. Panchuk RR, Prylutska SV, Chumakl VV, et al. Application of C60 fullerene-doxorubicin complex for tumor cell treatment in vitro and in vivo. J Biomed Nanotechnol. 2015;11:1139-1152.

10. Joshi M, Kumar P, Kumar R, et al. Aminated carbon-based "cargo vehicles" for improved delivery of methotrexate to breast cancer cells. Mater Sci Eng C Mater Biol Appl. 2017;75:1376-1388.

11. Prylutska SV, Skivka LM, Didenko GV, et al. C60 fullerene enhances cisplatin anticancer activity and overcomes tumor cell drug resistance. Nano Res. 2017;10:652-671.

12. Prylutska SV, Skivka LM, Didenko GV, et al. Complex of C60 fullerene with doxorubicin as a promising agent in antitumor therapy. Nanoscale Res Lett. 2015;10(1):499.

13. Samanta PN, Das KK. Noncovalent interaction assisted fullerene for the transportation of some brain anticancer drugs: a theoretical study. J Mol Graph Model. 2017;72:187-200.
14. Dugan LL, Turetsky DM, Du C, et al. Carboxyfullerenes as neuroprotective agents. Proc Natl Acad Sci U S A. 1997;94(17):9434-9439.

15. Lu T, Kao P, Lee C, Huang S, Lin C. C60 fullerene nanoparticle prevents $\beta$-amyloid peptide induced cytotoxicity in neuro 2A cells. J Food Drug Anal. 2011;19:151-158.

16. Prylutska SV, Burlaka AP, Matyshevska OP, et al. Effect of the visible light irradiation of fullerene-containing composites on the ROS generation and the viability of tumor cells. Exp Oncol. 2006;28(2): $160-162$.

17. Davydenko MO, Radchenko EO, Yashchuk VM, et al. Sensibilization of fullerene $\mathrm{C}_{60}$ immobilized at silica nanoparticles for cancer photodynamic therapy. J Mol Liq. 2006;127:145-147.

18. Scharff P, Ritter U, Matyshevska OP, et al. Therapeutic reactive oxygen generation. Tumori. 2008;94:278-283.

19. Sayes CM, Fortner JD, Guo W, et al. The differential cytotoxicity of water-soluble fullerenes. Nano Lett. 2004;4:1881-1887.

20. Foley S, Crowley C, Smaihi M, et al. Cellular localisation of a watersoluble fullerene derivative. Biochem Biophys Res Commun. 2002; 294:116-119.

21. Raoof M, Mackevey Y, Cheney MA, Wilson LJ, Curley SA. Internalization of $\mathrm{C} 60$ fullerenes into cancer cells with accumulation in the nucleus via the nuclear pore complex. Biomaterials. 2012;33(10):2952-2960.

22. Prylutska S, Bilyy R, Overchuk M, et al. Water-soluble pristine fullerenes C60 increase the specific conductivity and capacity of lipid model membrane and form the channels in cellular plasma membrane. J Biomed Nanotechnol. 2012;8(3):522-527.

23. Prylutskyy Y, Bychko A, Sokolova V, et al. Interaction of C60 fullerene complexed to doxorubicin with model bilipid membranes and its uptake by HeLa cells. Mater Sci Eng C. 2016;59:398-403.

24. Misra C, Thotakura N, Kumar R, et al. Improved cellular uptake, enhanced efficacy and promising pharmacokinetic profile of docetaxel employing glycine-tethered C60-fullerenes. Mater Sci Eng C. 2017;76:501-508.

25. Huang F, Mackeyev Y, Watson E, Cheney MA, Wilson LJ, Suh J. Evidence for nuclear internalisation of biocompatible [60]fullerene. Eur J Nanomed. 2013;5:51-55.

26. Prylutska SV, Matyshevska OP, Golub AA, et al. Study of $\mathrm{C}_{60}$ fullerenes and $\mathrm{C}_{60}$-containing composites cytotoxicity in vitro. Mater Sci Eng C. 2007;27:1121-1124.

27. Tolkachov M, Sokolova V, Loza K, et al. Study of biocompatibility effect of nanocarbon particles on various cell types in vitro. Materwiss Werksttech. 2016;47:216-221.

28. Boltalina OV, Abdul-Sada AK, Taylor R. Hyperfluorination of [60] fullerene by krypton difluoride. J Chem Soc Perkin Trans. 1995; 2:981-985.

29. Kim M, Gillies RJ, Rejniak KA. Current advances in mathematical modeling of anti-cancer drug penetration into tumor tissues. Front Oncol. 2013;3:1-10.

30. Hak S, Reitan NK, Haraldseth O, De Lange Davies C. Intravital microscopy in window chambers: a unique tool to study tumor angiogenesis and delivery of nanoparticles. Angiogenesis. 2010;13(2):113-130.

31. Tozer GM, Ameer-Beg SM, Baker J, et al. Intravital imaging of tumour vascular networks using multi-photon fluorescence microscopy. $A d v$ Drug Deliv Rev. 2005;57(1):135-152.

32. Jain R. Delivery of molecular and cellular medicine to solid tumors. Adv Drug Deliv Rev. 2012;64(suppl):353-365.

33. Jain RK. Understanding barriers to drug delivery: high resolution in vivo imaging is key. (Editorial). Clin Cancer Res. 1999;5:1605-1606.

34. Smith BR, Kempen P, Bouley D, et al. Shape matters: intravital microscopy reveals surprising geometrical dependence for nanoparticles in tumor models of extravasation. Nano Lett. 2013;18:1199-1216.

35. Smith BR, Zavaleta C, Rosenberg J, et al. High-resolution, serial intravital microscopic imaging of nanoparticle delivery and targeting in a small animal tumor model. Nano Today. 2013;8. doi:10.1016/j.nantod.2013.02.004.

36. Cheng SH, Li FC, Souris JS, et al. Visualizing dynamics of sub-hepatic distribution of nanoparticles using intravital multiphoton fluorescence microscopy. ACS Nano. 2012;6(5):4122-4131. 
37. Thurber GM, Yang KS, Reiner T, et al. Single-cell and subcellular pharmacokinetic imaging allows insight into drug action in vivo. Nat Commun. 2013;4:1504.

38. Miller MA, Gadde S, Pfirschke C, et al. Predicting therapeutic nanomedicine efficacy using a companion magnetic resonance imaging nanoparticle. Sci Transl Med. 2015;7(314):314ra183.

39. Dreher MR, Liu W, Michelich CR, Dewhirst MW, Yuan F, Chilkoti A. Tumor vascular permeability, accumulation, and penetration of macromolecular drug carriers. J Natl Cancer Inst. 2006;98(5): 335-344.

40. Tong L, He W, Zhang Y, Zheng W, Cheng J-X. Visualizing systemic clearance and cellular level biodistribution of gold nanorods by intrinsic two-photon luminescence. Langmuir. 2009;25(21):12454-12459.

41. Wharton T, Kini VU, Mortis RA, Wilson LJ. New non-ionic, highly water-soluble derivatives of C60 designed for biological compatibility. Tetrahedron Lett. 2001;42:5159-5162.

42. van de Ven AL, Wu M, Lowengrub J, et al. Integrated intravital microscopy and mathematical modeling to optimize nanotherapeutics delivery to tumors. AIP Adv. 2012;2(1):11208.

43. Pawley JB. Handbook of Biological Confocal Microscopy. Handbook of Biological Confocal Microscopy. Berlin: Springer; 2006.

44. Berne BJ, Pecora R. Dynamic Light Scattering. With Applications to Chemstriy, Biology, and Physics. Hoboken, NJ: Wiley-Interscience; 1976.

45. Song T, Dai S, Tam K, Lee S, Goh S. Aggregation behavior of twoarm fullerene-containing poly(ethylene oxide). Polymer (Guildf). 2003;44:2529-2536.
46. Laus S, Sitharaman B, Tóth E, et al. Destroying gadofullerene aggregates by salt addition in aqueous solution of $\mathrm{Gd} @ \mathrm{C} 60(\mathrm{OH}) \mathrm{x}$ and $\mathrm{Gd} @$ C60[C(COOH 2)]10. J Am Chem Soc. 2005;127(26):9368-9369.

47. Petros RA, DeSimone JM. Strategies in the design of nanoparticles for therapeutic applications. Nat Rev Drug Discov. 2010;9(8):615-627.

48. Nie S. Understanding and overcoming major barriers in cancer nanomedicine (Editorial). Nanomedicine. 2010;5(4):523-528.

49. Hunter RJ. Introduction to Modern Colloid Science. Oxford: Oxford University Press; 1993.

50. Nagy JA, Benjamin L, Zeng H, Dvorak AM, Dvorak HF. Vascular permeability, vascular hyperpermeability and angiogenesis. Angiogenesis. 2008;11(2):109-119.

51. Dellian M, Yuan F, Trubetskoy VS, Torchilin VP, Jain RK. Vascular permeability in a human tumour xenograft: molecular charge dependence. Br J Cancer. 2000;82:1513-1518.

52. Yuan F, Dellian M, Fukumura D, et al. Vascular permeability in a human tumor xenograft: molecular size dependence and cutoff size advances in brief vascular permeability in a human tumor xenograft: molecular size dependence and cutoff size1. Cancer Res. 1995;55:3752-3756.

53. Pearce ML, Lewis AE, Kaplan MR. The Factors Influencing Circulation Time. Circulation. 1952;5:583-588.

54. Janssen B, Debets J, Leenders P, Smits J. Chronic measurement of cardiac output in conscious mice. Am J Physiol Regul Integr Comp Physiol. 2002;282(3):R928-R935.

55. Kreissl MC, Wu HM, Stout DB, et al. Noninvasive measurement of cardiovascular function in mice with high-temporal-resolution smallanimal PET. J Nucl Med. 2006;47(6):974-980. 


\section{Supplementary materials}

Materials and methods

HPLC analysis of $\mathrm{C}_{60}$-serPF, PF dye and HeLa cell homogenate

Separation chromatography of $\mathrm{C}_{60}$-serPF was performed using Waters Acquity UPLC I class system (Milford, MA, USA) comprising a quaternary solvent manager, equipped with flow through needle autosampler, fluorescence detector and column manager using two Acquity BEH C18 Columns, consecutively connected, $130 \AA$, $1.7 \mu \mathrm{m}, 3.0 \times 100 \mathrm{~mm}$ column with an BEH C18 $1.7 \mu \mathrm{m}$ VanGuard pre-column $(2.1 \times 5 \mathrm{~mm})$ and Waters fractions collector III. The columns were re-equilibrated with initial conditions for $5 \mathrm{~min}$. Columns temperature was maintained at $30^{\circ} \mathrm{C}$. Excitation of fluorescence detector was set at $630 \mathrm{~nm}$, emission recorded at $666 \mathrm{~nm}$. Mobile phase compositions: $\mathrm{A}=$ ammonium formate/formic acid buffer at $0.015 \mathrm{M}$ with $\mathrm{pH}=3.8, \mathrm{~B}=$ methanol, $\mathrm{C}=$ acetonitrile, $\mathrm{D}=$ acetic acid/acetonitrile at 1:9 ratio. Gradients were applied linearly as follows:
$0 \min -99 \% \mathrm{~A}, 1 \% \mathrm{D}$ at $0.25 \mathrm{~mL} / \mathrm{min} ; 2 \min -90 \% \mathrm{~A}$, $9 \% \mathrm{~B}, 1 \% \mathrm{D}$ at $0.25 \mathrm{~mL} / \mathrm{min} ; 14 \mathrm{~min}-65 \% \mathrm{~A}, 25 \% \mathrm{~B}, 9 \%$ $\mathrm{C}, 1 \% \mathrm{D}$ at $0.2 \mathrm{~mL} / \mathrm{min} ; 21 \mathrm{~min}-90 \% \mathrm{~B}, 9 \% \mathrm{C}, 1 \% \mathrm{D}$ at 0.15 $\mathrm{mL} / \mathrm{min} ; 24 \mathrm{~min}-99 \% \mathrm{~A}, 1 \% \mathrm{D}$ at $0.2 \mathrm{~mL} / \mathrm{min}$. The maximum flow-rate error was $\pm 2 \%$ over the gradient range.

\section{Exposure of HeLa cells to $\mathrm{C}_{60}$-serPF and preparation} of homogenate for HPLC

HeLa cervical cancer cells (ATCC, Manassas, VA, USA) were incubated with $\mathrm{C}_{60}$-serPF (at $1 \mathrm{mg} / \mathrm{mL}$, in PBS) for $24 \mathrm{~h}$ using 96-well plate. On the following day the cells $\left(\sim 10^{6}\right)$ were pelleted, washed twice in phosphate-buffered saline, and suspended in $2 \mathrm{~mL}$ of $1 \%$ Triton X-100 in glass vial, homogenized by repeated uptake into a pipette tip and then sonicated using bath sonicator for $20 \mathrm{~min}$. After centrifugation at $10,000 \times \mathrm{g}$ for $20 \mathrm{~min}$ at RT, the supernatant was subjected to $0.05 \mathrm{~mm}$ filtration using $0.05 \mathrm{~mm}$ Anotop 10 Plus syringe filter (Sigma-Aldrich, St Louis, MO, USA), and resulting filtrate analyzed by HPLC.
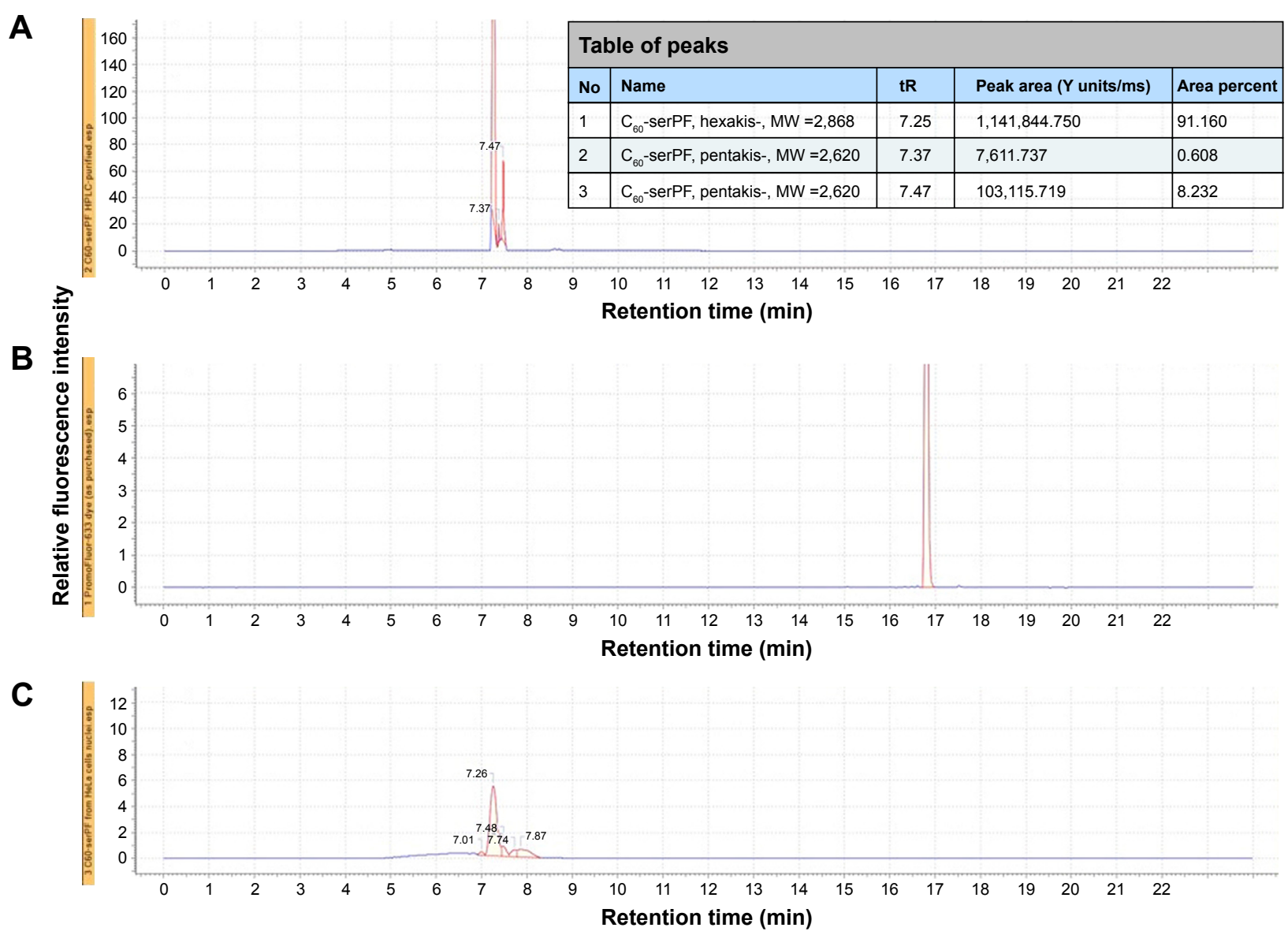

Figure SI HPLC separation chromatograms of (A) $\mathrm{C}_{60}$-serPF, (B) free PromoFluor-633 (PF) dye and (C) homogenate of $\mathrm{C}_{60}$-serPF incubated with HeLa cervical cancer cells showing relative fluorescence intensity versus retention time $(\mathrm{tR})$, min.

Notes: For fluorescence detection, PF dye was excited at $630 \mathrm{~nm}$, emission at $666 \mathrm{~nm}$. The inset table shows peak areas and relative percentage for each of the adducts. Total peak area includes $4 \%$ water. 


\section{Results}

In Figure S2, Panel A, the HPLC separation chromatogram $\mathrm{C}_{60}$-serPF shows only one group of bands between 7 and 8 min retention time. The slight difference in retention times is due to the presence of hexakis-substituted [60]fullerene, (of the expected $\mathrm{C}_{60}$-serPF structure) and a small admixture of pentakis-adduct. Further chromatographic separation of these two almost identical [60]fullerene derivatives is difficult. The $\mathrm{C}_{60}$-serPF product consists of $90 \% \pm 2.0 \%$ of hexakis-adduct, $6 \% \pm 2.0 \%$ of pentakis-adduct, subtracting out $\sim 4 \%$ water due to $\mathrm{C}_{60}$-ser hygroscopicity.

Figure S2, Panel B shows the HPLC chromatogram of the free $\mathrm{PF}$ dye, with a single band and a retention time nearly double that of $\mathrm{C}_{60}$-serPF. The absence of this band in Panel A, demonstrates that the PF dye, which is covalently bound to the fullerene does not detach from the fullerene, nor is it present after purification of the conjugate. To demonstrate that $\mathrm{C}_{60}$-serPF does not undergo hydrolysis by cell cytoplasm, HeLa cervical cancer cells were incubated with $\mathrm{C}_{60}$-serPF (at $1 \mathrm{mg} / \mathrm{mL}$ in PBS) for $24 \mathrm{~h}$ using 96-well plate. The next day the cells $\left(10^{6}\right.$ in average) were pelleted, homogenized, and the resulting filtrate analyzed by HPLC, Figure S2, Panel C. The chromatogram of $\mathrm{C}_{60}$-serPF extracted from cells shows the same band positions as the original $\mathrm{C}_{60}$-serPF with no band present from the PF dye. Additionally, $\mathrm{C}_{60}$-serPF has been tested in $0.1 \mathrm{M}$ and $1 \mathrm{M}$ acetic acid solutions, and porcine liver esterase (Sigma-Aldrich) in $0.1 \mathrm{M}$ phosphate buffer (pH 6.8) with the same result in each case.

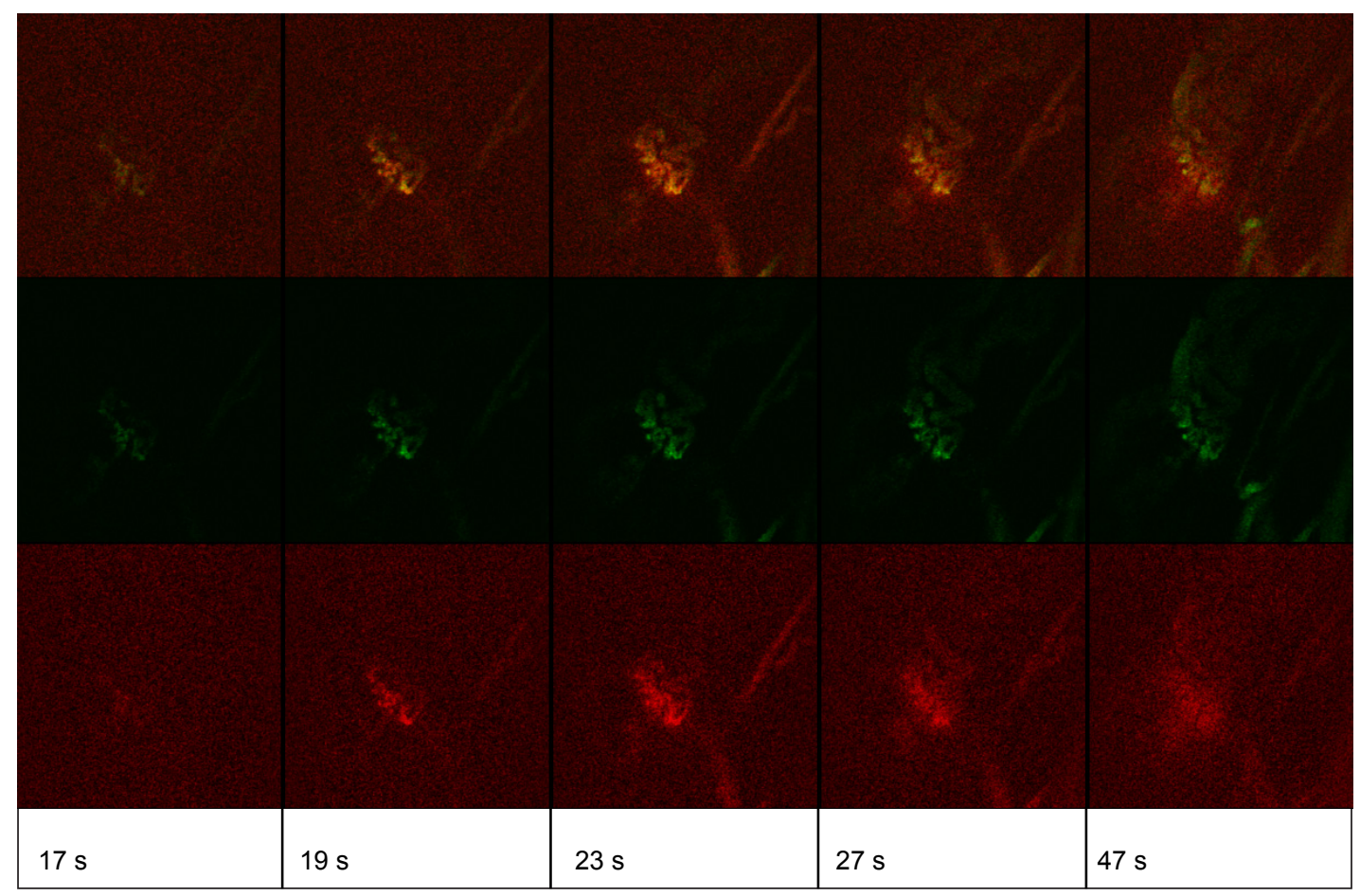

Figure S2 IVM image series and fluorescence quantification of $\mathrm{C}_{60}$-serPF delivery to tumor microvasculature, with fluorescence intensity in intravascular ROI not saturated. Unadjusted video images.

Notes: IVM video images of $\mathrm{C}_{60}$-serPF and FITC-Dextran co-injected in channels Cy5, FITC and merged (bottom, middle and top rows respectively) collected at I6X magnification at $2.5 \mathrm{fps}$ using a Resonant scanner in mouse \#2. Select times shown post-injection.

Abbreviations: IVM, intravital microscopy; ROI, regions of interest.

International Journal of Nanomedicine

\section{Publish your work in this journal}

The International Journal of Nanomedicine is an international, peerreviewed journal focusing on the application of nanotechnology in diagnostics, therapeutics, and drug delivery systems throughout the biomedical field. This journal is indexed on PubMed Central, MedLine, CAS, SciSearch ${ }^{\circledR}$, Current Contents ${ } /$ Clinical Medicine,

\section{Dovepress}

Journal Citation Reports/Science Edition, EMBase, Scopus and the Elsevier Bibliographic databases. The manuscript management system is completely online and includes a very quick and fair peer-review system, which is all easy to use. Visit http://www.dovepress.com/ testimonials.php to read real quotes from published authors.

\footnotetext{
Submit your manuscript here: http://www.dovepress.com/international-journal-of-nanomedicine-journal
} 